\title{
Ion Energy Diagnostics in the Plume of an SPT-100 from Thrust Axis to Backflow Region
}

\author{
Lyon B. King ${ }^{*}$ and Alec D. Gallimore \\ Plasmadynamics and Electric Propulsion Laboratory \\ Department of Aerospace Engineering \\ University of Michigan \\ Ann Arbor, MI USA
}

\begin{abstract}
Of primary concern with the integration of Hall thrusters on conventional satellite designs is the possible damaging effect of high-energy exhaust ions impinging upon spacecraft surfaces. This paper reports on measurements of plasma ion energy distributions within the plume of an SPT-100 Hall thruster using a custom-designed molecular beam mass spectrometer. With this instrument ion energy was measured over a complete 360-degree circumference about the thruster at $0.5-\mathrm{m}$ radius from the exit plane and over a total inclusive arc of 260 degrees at $1.0-\mathrm{m}$ radius. These data uncovered the existence of high-energy ions departing the thruster at angles exceeding 90 degrees from the thrust vector and continuing well into the backflow region of the plume. Through an analysis of the energy structure the evidence of charge-exchange collisions occurring between plume ions and background neutrals was documented; such collisions produced anomalous distributions of ions having voltages greater than that applied to the thruster discharge.
\end{abstract}

\section{Nomenclature}

$\begin{array}{ll}\mathrm{y} & \text { Spatial coordinate }(\mathrm{m}) \\ \mathrm{x} & \text { Spatial coordinate }(\mathrm{m}) \\ \mathrm{d} & \text { Analyzer plate separation }(\mathrm{m}) \\ \mathrm{l} & \text { Analyzer interslit distance }(\mathrm{m}) \\ \mathrm{w} & \text { Analyzer slit width }(\mathrm{m}) \\ \mathrm{V}_{\mathrm{p}} & \text { Repelling plate voltage }(\mathrm{V}) \\ \mathrm{q} & \text { Ion integer charge state } \\ \mathrm{e} & \text { Elementary charge (C) } \\ \mathrm{m}_{\mathrm{i}} & \text { Mass of ion (kg) } \\ \mathrm{ui} & \text { Ion velocity (m/s) } \\ \theta & \text { Angle (deg) } \\ \mathrm{E}_{\mathrm{i}} & \text { Ion energy (J or eV) } \\ \mathrm{K}_{45} & \text { Spectrometer constant } \\ \mathrm{V}_{\mathrm{i}} & \text { Ion voltage (V) } \\ \mathrm{G}_{\mathrm{CEM}} & \text { Gain of electron multiplier }\end{array}$

A. Area of collector $\left(\mathrm{m}^{2}\right)$

$\mathrm{n}_{\mathrm{i}} \quad$ Ion density $\left(\mathrm{m}^{-3}\right)$

$\mathrm{f}\left(\mathrm{V}_{\mathrm{i}}\right)$ Ion voltage distribution $(\mathrm{s} / \mathrm{m})$

$r \quad$ Radial distance from thruster $(\mathrm{m})$

$\mathrm{f}\left(\mathrm{u}_{\mathrm{i}}\right) \quad$ Ion velocity distribution $(\mathrm{s} / \mathrm{m})$

$\beta \quad$ Maxwellian constant $(\mathrm{m} / 2 \mathrm{kT})$

$\mathrm{u}_{\mathrm{d}} \quad$ Bulk drift velocity $(\mathrm{m} / \mathrm{s})$

$\mathrm{k} \quad$ Boltzmann constant $(\mathrm{J} / \mathrm{K})$

$\mathrm{T}_{\mathrm{i}} \quad$ Ion temperature (K)

$\mathrm{T}_{\mathrm{eV}} \quad$ Ion temperature (eV)

$\tau_{\mathrm{i}} \quad$ Defined ion temperature $(\mathrm{V})$

$\mathrm{T}_{\mathrm{e}} \quad$ Electron temperature $(\mathrm{eV})$

$\mathrm{V}_{\mathrm{m}} \quad$ Most-probable voltage (V)

$\mathrm{V}_{\mathrm{b}} \quad$ Main distribution voltage (V)

Copyright (C) 1998 by Lyon B. King. Printed by the American Institute of Aeronautics and Astronautics, Inc. with permission.

* Research Associate, Member AIAA. Current address: NIST, Time \& Frequency Div. 847, 325 Broadway, Boulder, CO 80303

\# Associate Professor, Senior member AIAA 


\section{Introduction}

Of primary concern with the use of Hall thrusters on geostationary communications satellites is the possible damaging effect of the highly energetic plasma exhaust plume on spacecraft surfaces. Specific issues include the erosion of solar array material due to incident high energy propellant ions, coating and contamination of solar arrays and other surfaces due to efflux of thruster self-erosion material, heating of sensitive spacecraft components, and uneven spacecraft charging due to impinging propellant ions. In order to fully quantify and understand these phenomena extensive characterization of the plasma plume has been performed over the past few years.

The initial effort towards characterizing the Hall thruster plume was undertaken by a joint industry team comprised of Russian and U.S. researchers. ${ }^{1}$ This test utilized a set of sample slides representative of spacecraft surfaces placed in the plume flow to model erosion and contamination in addition to the use of a Faraday probe to measure ion current density and a gridded Retarding Potential Analyzer (RPA) to measure the ion energy distribution. Due to the rapid decay in ion density with increasing distance from the thruster centerline (thrust vector), these diagnostics were limited to points lying within 60 degrees of the thrust vector. Within this volume, however, the RPA data demonstrated some intriguing trends: although the thruster discharge voltage was set to $300 \mathrm{~V}$, the ion energy distribution curve showed a significant "tail" representing ions accelerated through potentials as great as 350 to $450 \mathrm{~V}$. Possible reasons for the existence of this anomalous high-energy population were not addressed. More plume characterization testing followed: Myers and Manzella performed additional measurements using an RPA, but the data was limited to very few spatial locations within the plume and again was confined to points within 60 degrees of the axis. ${ }^{2}$ These data, as well, suggested the existence of ions with voltages greater than that supplied by the applied discharge. The region of the plume with the greatest interest for spacecraft designers was the far off-axis region (angles greater than 60 degrees from centerline) due to the probable location of spacecraft surfaces. The ion current density in this region (out to 100 degrees) was probed by Manzella and Sankovic, ${ }^{3}$ however no ion energy diagnostics have been performed out to such large angles.

Continued studies of plume-induced erosion and contamination were performed utilizing more extensive test matrices comprising a wide array of representative spacecraft materials exposed to a large volume of the plasma plume, including the far off-axis region. ${ }^{4,5}$ For the most part, these studies were addressed not at deepening the knowledge of the plasma properties within the plume, instead they documented the erosion/contamination problem from a top-level perspective by simply measuring the net effects of the plume on representative spacecraft materials. For near-term applications of the SPT-100 these top-level studies provided sufficient data bases to enable integration of the Hall thruster with western satellites. However, in order to prevent the need for further extensive sample testing to accommodate new spacecraft materials or design configurations in the future, it was apparent that a more thorough understanding of the underlying physics and properties of the plasma plume was required.

This paper represents one part of a comprehensive study performed at the University of Michigan to characterize the heavy-particle plasma properties within the Hall thruster plume over a large volume in space including the far-off axis regions as well as the backflow area directly behind the thruster. The purpose of this paper is to present 
measurements of the ion energy acquired through the use of a custom-designed molecular beam mass spectrometer (MBMS) for Hall thruster research.

\section{Description of Apparatus}

The MBMS system used a set of orifice skimmers to admit a beam of plume ions from the main vacuum chamber into an array of differentially pumped sub-chambers. The sub-chambers were maintained at high vacuum to minimize and effectively eliminate collisions involving ions within the beam. A sampling skimmer orifice was mounted on the upstream end of the MBMS; this orifice skimmed off a small diameter ion beam into the first sub-chamber. This beam was then collimated by a second orifice at the downstream end of the first sub-chamber. The collimated beam then passed through the entrance slit of a 45-degree electrostatic energy analyzer. This analyzer employed a constant electric field such that only ions with a pre-selected energy have a trajectory which permits them to traverse the exit slit and impinge upon a detector. Ion mass detection was accomplished through a time-of-flight method. The mass diagnostics are the subject of a related paper presented at the same conference. ${ }^{6}$ This paper will focus on the global (species independent) ion energy distribution.

The 45-degree electrostatic energy analyzer is a flexible, robust method for particle energy filtering that has been used widely in beam physics research. ${ }^{7,8,9}$ A schematic of the system utilized in the MBMS is shown in Figure 1 with coordinate system and relevant dimensions defined. The ion beam is admitted through the entrance slit of the analyzer and immediately enters a region of constant electric field of magnitude $\mathrm{V}_{\mathrm{p}} / \mathrm{d}$ oriented at an angle $\theta$ to the direction of travel. The ions thus experience a constant acceleration in the negative $y$-direction such that the spatial equation of their trajectory is

Eqn. 1

$$
y=x-\frac{q_{i} e V_{p}}{2 d m_{i}} \frac{x^{2}}{u_{i}^{2} \sin ^{2} \theta}
$$

$$
\text { Since } \theta=45 \text { degrees and } u_{i}^{2}=2 E_{i} / m_{i} \text { Eqn. } 1 \text { becomes }
$$

Eqn. 2

$$
\left.y=x-\frac{1}{2 d} \frac{V_{p}}{\left(E_{i} / q_{i} e\right.}\right) x^{2}
$$

In order for an ion to pass through the analyzer and escape through the exit slit to the detector it must intersect the point $y=0, x=1$; this pass constraint is defined as the spectrometer constant, $\mathrm{K}_{45}$, and is given by

Eqn. 3

$$
\mathrm{K}_{45} \equiv \frac{\mathrm{V}_{\mathrm{p}}}{\left(\mathrm{E}_{\mathrm{i}} / \mathrm{q}_{\mathrm{i}} \mathrm{e}\right)}=\frac{2 \mathrm{~d}}{1} \text {. }
$$

The analyzer thus performs the function of an energy-per-charge filter, $\mathrm{E}_{\mathrm{i}} / \mathrm{q}_{\mathrm{i}} \mathrm{e}$. Since the beam ions within the Hall thruster plume experienced a discharge acceleration according to $\mathrm{q}_{\mathrm{i}} \mathrm{eV}_{\mathrm{i}}=1 / 2 \mathrm{~m}_{\mathrm{i}} \mathrm{u}_{\mathrm{i}}^{2}$, the value of energy-per-charge for an ion is equivalent to the acceleration voltage, $\mathrm{V}_{\mathrm{i}}$. For a given value of repelling plate voltage, only ions with 
Eqn. 4

$$
\mathrm{V}_{\mathrm{i}}=\frac{1 / 2 \mathrm{~m}_{\mathrm{i}} \mathrm{u}_{\mathrm{i}}^{2}}{\mathrm{q}_{\mathrm{i}} \mathrm{e}}=\frac{\mathrm{V}_{\mathrm{p}}}{\mathrm{K}_{45}}
$$

will reach the collector and be recorded as ion current.

The current detector employed by the MBMS was a ceramic channel electron multiplier (CEM) capable of amplifying the input ion current by a factor greater than $1 \times 10^{8}$ (K-M Electronics model $7550 \mathrm{~m}$ ) with a maximum output current of approximately $5 \mu \mathrm{A}$. An important consideration was necessary when using the CEM in a multi-component ion beam: the initial "charge event" starting the electron cascade was due to secondary electrons ejected from an ion-wall collision. The number of electrons ejected per ion impact is a function of the material properties of the channel coating. Therefore an ion of charge $\mathrm{q}=2$ does not cause twice as much electron current as a singly charged ion (as is the case in a conducting metal collector such as an electrostatic probe). Therefore the CEM serves essentially as an ion counter rather than a charge counter.

For a single species flow the 45-degree electrostatic analyzer technique produces an ion current vs. repelling voltage trace which is directly proportional to the ion energy distribution function, analogous to the RPA technique. However, unlike the RPA technique, the 45-degree analyzer requires no numerical differentiation of raw data to obtain the distribution and the resultant curves are therefore much more precise and smooth. Unfortunately, like the RPA, the existence of multiple ion species in the beam complicates interpretation of the data. This can be demonstrated by analyzing the output of the CEM. Since the CEM produced a current proportional to the number of ions incident on the collector, the current output can be written as

Eqn. 5

$$
I_{j}=G_{C E M} A_{c} n_{i}\left\langle u_{i}\right\rangle \text {. }
$$

According to Eqn. 4 only ions with a discrete voltage, $V_{i}$, will be detected by the CEM such that

Eqn. 6

$$
\left\langle u_{i}\right\rangle=\left\langle\sqrt{\frac{2 q_{i} e_{i}}{m_{i}}}\right\rangle=\sqrt{\frac{2 q_{i} \mathrm{eV}_{i}}{m_{i}}}
$$

so that now the current output of the CEM for a single value of ion voltage is written as

Eqn. 7

$$
I_{i}\left(V_{i}\right)=G_{C E M} A_{c} n_{i}\left(V_{i}\right) \sqrt{\frac{2 q_{i} e V_{i}}{m_{i}}}
$$

where $n_{i}\left(V_{i}\right)$ is the number density of ions with voltage $V_{i}$ which is precisely the ion voltage distribution function, $n_{i}\left(V_{i}\right)=f\left(V_{i}\right)=f\left(E_{i} / q_{i} e\right)$. Realizing this fact it is apparent from Eqn.7 that the ion current vs. voltage is not directly proportional to the ion energy distribution function as is widely accepted with these devices. Rather, in a multi-component ion beam the 45-degree energy analyzer yields data which is related to the voltage distribution function according to

Eqn. 8

$$
f\left(V_{i}\right) \propto \frac{I_{i}\left(V_{i}\right)}{\sqrt{q_{i} e V_{i}}}
$$


Even with the complications imposed on the data interpretation scheme by the effects of a multi-component flow, the 45-degree electrostatic energy analyzer yields great insight to the ion dynamics. Of considerable interest to this research is the ion voltage: by analyzing the ion voltage distribution information is gained regarding the acceleration of ions within the thruster discharge chamber. Eqn. 8 shows that by dividing the recorded ion current by the square root of the ion pass voltage a function very nearly equal to $f\left(V_{i}\right)$ is obtained; the existence of multiply charged ions only weakly affects the interpretation of these data for most flows. For example, the Hall thruster plasma has been estimated to consist of roughly $10 \%$ ions with $q=2$. The results of Eqn. 8 would then dictate that for a given value of measured ion current, a $10 \%$ fraction of the total current magnitude should be moderated by a factor of 0.707 (corresponding to $2^{-1 / 2}$ ). This constitutes a very small correction and thus, the measured ion current vs. voltage curve is very nearly equal to the voltage distribution function, $f\left(V_{i}\right)$.

The 45-degree electrostatic energy analyzer was constructed of 1.5 -mm-thick aluminum plates. In order to eliminate field distortion within the analyzer due to the surrounding ground potential of the vacuum chamber walls and to ensure a homogeneous electric field a set of seven centrally slotted field correction plates were mounted intermediate to the repelling plate and the entrance ground plate. These correction plates were biased using a resistor string voltage divider to force the field equipotentials at the mid-planes and minimize field leakage. The entire plate system was supported on a frame constructed of 3/16-inch-diameter nylon threaded rods to ensure electrical isolation of each plate. given by

The resolving power of the analyzer is dictated by geometric parameters and is

Eqn. 9

$$
\frac{\Delta \mathrm{V}_{\mathrm{i}}}{\mathrm{V}_{\mathrm{i}}}=\frac{\mathrm{w} \sin \theta}{\mathrm{l}}
$$

The desired voltage resolution was used to select the proper values for $l$ and $w$. The depth of the analyzer, $\mathrm{d}$, was then dictated by the parabolic trajectory of the ions which pass through the analyzer to the exit slit: the apex of this trajectory must not intersect the repelling back plate. Manipulation of Eqn. 2 yields the necessary condition that $\mathrm{d}>l / 4$. The pertinent parameters of the 45-degree electrostatic energy analyzer used in the MBMS for this research are presented in Table 1.

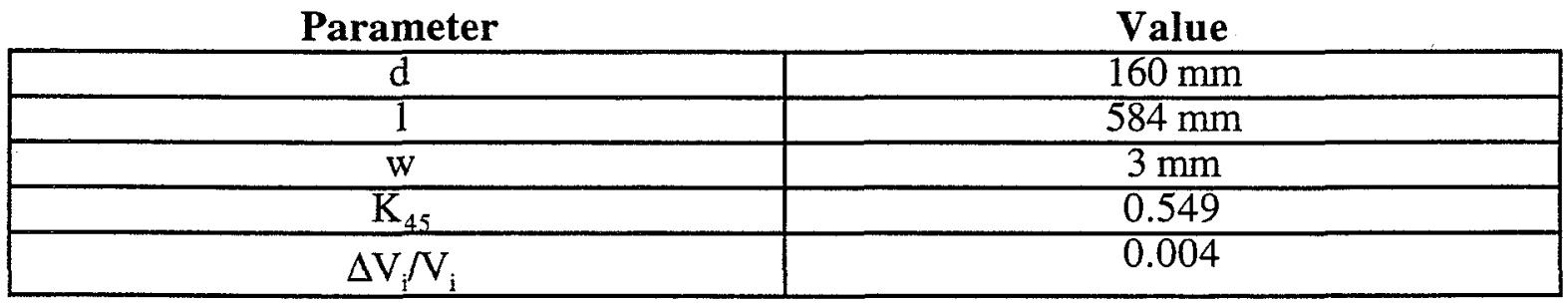

Table 1. Physical characteristics and resolving power of 45-degree electrostatic energy analyzer. 


\section{Experimental Set-up}

The SPT-100 was mounted to a rotary table such that the rotation axis coincided with the center of the exit plane of the thruster. Therefore, by rotating the thruster relative to the fixed MBMS skimmer inlet the plasma plume could be sampled as a function of angular position at a fixed radial distance, $r$, from the exit plane. This set-up is illustrated schematically in Figure 2. The centerline (thrust axis) of the thruster was denoted as zero degrees, with positive theta values representing points in the cathode half-plane of rotation (the angular position shown in Figure 2 represents $\theta=+90$ degrees). The angular alignment of the thruster and MBMS was achieved by using a laboratory laser to establish the MBMS beam line. The laser beam line was used to verify the angular orientation of the 45-degree electrostatic analyzer to within 0.5 degrees; similarly, the thruster was rotated such that the laser beam line was precisely aligned with the center of the exit plane of the thruster, as shown in Figure 2, establishing the 90 degree position of the SPT-100 to better than 0.5 degrees. Since the relative uncertainty in angular position of the rotary table was 0.1 degrees, the uncertainty in position for all data points is,+-0.5 degrees due to initial alignment uncertainty. By re-locating the rotary table mount between tests, data were obtained as a function of angular position for radial distances from the thruster of $\mathrm{r}=0.5 \mathrm{~m}$ and $\mathrm{r}=1.0 \mathrm{~m}$.

The 45-degree analyzer repelling voltage was supplied by slowly varying the output of a high-precision sourcemeter (Keithley 2410). The sourcemeter provided regulated voltage with better than $0.012 \%$ accuracy over a range of zero to $1100 \mathrm{~V}$. The CEM current was measured with a sensitive picoammeter (Keithley 486) and recorded as a function of 45-degree analyzer pass voltage. By utilizing the picoammeter, the high-gain $\mathrm{CEM}$, and long sampling times true ion currents as low as $5 \times 10^{-19}$ A could be accurately measured; this represents an ion flux on the order of 1 ion per second incident on the CEM.

\section{Ion Voltage Measurements in an SPT-100 at $0.5 \mathrm{~m}$}

The ion current incident on the CEM was recorded as a function of ion voltage by varying the repelling potential on the 45-degree energy analyzer. In this fashion curves were obtained at a radial distance of $0.5 \mathrm{~m}$ from the thruster exit plane as a function of angular position about the thrust axis in 10-degree intervals. The high-gain attributed to the picoammeter and CEM enabled data to be obtained in a complete 360-degree envelope about the SPT-100. Peak ion current values fell as low as $1 \times 10^{-18} \mathrm{~A}$ for points directly behind the thruster. These sweeps are shown in Figure 3 through Figure 11. The abscissa of the ion current curves have been corrected for the energy imparted to the ions as they fell from ambient plasma potential through the skimmer inlet to ground potential; the magnitude of the required correction was measured using a Langmuir probe immediately upstream of the sampling skimmer to interrogate the local plasma potential.

\section{Ion Voltage Measurements in an SPT-100 at $1.0 \mathrm{~m}$}

The ion energy distribution function was evaluated at a radial distance of $1.0 \mathrm{~m}$ from the thruster exit plane by repositioning the thruster/rotary table mount relative to the MBMS inlet skimmer. At this distance curves were obtained as a function of angular position about the SPT-100 thrust axis. Due to the much lower ion densities at $1.0 \mathrm{~m}$ as compared with those at $0.5 \mathrm{~m}$, data could not be obtained in a complete 360-degree arc 
about the thruster. The region behind the thruster at positive angles greater than 110 degrees and negative angles of magnitude greater than -150 degrees represented ion currents less than $5 \times 10^{-19} \mathrm{~A}$; due to the low currents this region could not be evaluated. The resulting data are shown in Figure 12 through Figure 18. These curves have been corrected for the parasitic energy addition imposed to the ions as they fell from local plasma potential through the inlet skimmer to ground potential.

The ion current traces obtained for the points at 10 degrees and -10 degrees were strikingly dissimilar to the overall trends exhibited as a function of angular position. This contrast is easily seen by examining Figure 12 and Figure 15. In order to more fully interrogate this region of the plume data were obtained with much finer angular resolution for points within 20 degrees of the axis. Figure 19 illustrates the overall trend in ion current between the thrust axis and 20 degrees, with Figure 20 included as an expanded view of the evolution between 6 degrees and 17 degrees. Figure 21 and Figure 22 present the ion current evolution for the positions between -5 degrees and -20 degrees.

\section{Discussion of Ion Voltage Distributions}

The relation linking the I(V) curve to the ion voltage distribution function was derived previously: as evidenced by Eqn. 8 calculation of the voltage distribution in a multispecies flow requires knowledge of the ionization-state-dependent current as a function of voltage. Specifically,

Eqn. 10

$$
f(V) \propto \frac{1}{\sqrt{V}}\left(\frac{I\left(V, q_{1}\right)}{\sqrt{q_{1}}}+\frac{I\left(V, q_{2}\right)}{\sqrt{q_{2}}}+\ldots\right)
$$

where $I\left(V, q_{n}\right)$ denotes the current due to ions with voltage $V$ and charge $q_{n}$. Since ion velocity increases with $\mathrm{q}$, a population of high-q ions will produce a larger current than an equal number of low-q ions: The inclusion of $\mathrm{q}^{-1 / 2}$ in Eqn. 10 is therefore required to account for the disproportional contribution of high-q ions to the total ion current. The data reported in Section 5 and 6 reflect the total current due to all ion charge states as a function of ion voltage, therefore they cannot be directly manipulated to yield $f(V)$. If the flow under consideration was composed almost entirely of one species of ion $(q=1)$ then it would be possible to obtain a good approximation of $f(V)$ by neglecting the contribution due to the higher charge states. This approximation has previously been applied to the RPA technique allowing valuable estimations of ion density and velocity to be made in the region of the plume near thruster centerline.

Although the $\mathrm{I}(\mathrm{V})$ curve is not directly proportional to $\mathrm{f}(\mathrm{V})$ for reasons discussed above, it should be kept in mind that these two functions are very closely related. For example, if a portion of the I(V) curve was known to result entirely from ions with $q=2$, attenuation of this portion of the curve by a factor of 0.707 would yield the value of $f(V)$. Allowing for the existence of ions with charge states up to $\mathrm{q}=4$ in the flow ensures that the value of $I(V)$ is never more than a factor of two larger than $f(V)$. Therefore, although it may be inappropriate to substitute $I(V)$ for $f(V)$ in detailed calculations, when discussing the overall shape and data trends it is reasonable to speak of the two functions interchangeably. 


\subsection{Comparison with RPA}

A cursory examination of the I(V) traces presented in Section 5 and 6 reveal a much improved resolution over the widely used RPA technique. The RPA data reduction process required numerical differentiation and hence produced very noisy distribution curves. The inherently different 45-degree analyzer technique required no differentiation and therefore produced much smoother, more accurate results. Additionally, the uncertainties associated with measuring the ion current in the RPA experiment combined with the noisy differentiation yielded data with unacceptable signal-to-noise ratios for angular positions exceeding 60 degrees from the thrust axis. The high gain of the CEM/picoammeter detector circuitry enabled measurement of the ion energy at points up to 180 degrees off thrust axis (directly behind the thruster). These far off-axis regions of the plume are especially critical in evaluating plume impingement on surrounding spacecraft surfaces.

A direct comparison between the MBMS and RPA data demonstrates a disagreement in the measured ion energy. Figure 23 shows a comparison of the two techniques at $0.5 \mathrm{~m}$ radius, while Figure 24 represents the points at $1.0 \mathrm{~m}$ radius. The difference between the MBMS-obtained energy distributions and the in-situ RPA probe data exposed a shortcoming in the probe technique as applied to a high-density, highvelocity plasma such as the Hall thruster plume. Physically, the RPA probe resembled a cylinder closed on one end by a current collector, with the open end exposed to the flowing plasma. The problem with this technique arises when the plasma flow velocity and density increase sufficiently to "choke" the closed internal volume of the probe. It was shown using a neutral particle flux probe that the internal pressure due to neutral Xe within the probe can exceed 10 or $20 \mathrm{~m}$ Torr in the SPT-100 plume near centerline at $0.5 \mathrm{~m} .{ }^{10}$ This stagnation pressure rise was due to the ram effect of the flowing high density plasma entering the probe and being neutralized by collisions with the probe walls or current collector forming a high-density trapped gas volume. This relatively dense target gas scatters the incoming plume ions through both charge exchange (CE) and momentum transfer collisions. Any collision involving a plume ion and a stagnant target particle results in energy loss for the plume ion; the net effect is an attenuation of the energy peak and a broadening of the distribution in the direction of lower ion energy.

Although detailed cross sections for all collisions likely to occur within the RPA volume were not available, an estimation of the effect of ram gas build-up was made based on those that were. For example, the dominant collision mechanism is likely to be $\mathrm{CE}$ between singly ionized xenon plume ions and background xenon neutrals; the cross section for this reaction can be calculated according to Reference 10. Assuming that the internal probe pressure is $20 \mathrm{mT}$ Torr with a temperature near that of the probe walls (300 $\mathrm{K}$ ), an incoming $300 \mathrm{~V} \mathrm{Xe}^{+}$ion experiences a CE mean free path on the order of $3 \mathrm{~mm}$. The RPA probe had a path length from inlet to collector of approximately $2 \mathrm{~cm}$ : this would cause over $90 \%$ of the plume ions to suffer a CE collision prior to detection. On the other hand, differential pumping allowed the MBMS to maintain an internal vacuum of approximately $1 \times 10^{-6}$ torr during operation; this equates to a mean-free-path on the order of $70 \mathrm{~m}$ for the CE collision. For ions traveling the $2.35 \mathrm{~m}$ path from the MBMS inlet to the detector this equates to a collision probability of approximately $3 \%$. It is therefore justified to assume that collisional broadening of the ion energy within the MBMS is negligible.

The collisional broadening and attenuation within the RPA should be greatest for regions of high density and high velocity. From an examination of Figure 23 and Figure 24 this is seen to be true. The energy shift between the RPA and MBMS data was of greatest magnitude for the near centerline traces where the ion density was highest. 
Furthermore, the shift in the near centerline data is more severe for the data taken at $0.5 \mathrm{~m}$ from the thruster than for the $1.0 \mathrm{~m}$ data set

\subsection{Ion Temperature}

Since the I(V) traces obtained with the 45-degree analyzer very closely approximate the ion voltage distribution function it is perhaps instructive to describe their shape in terms of an ion temperature. However, the driving mechanism defining the shape of the I(V) curves is an overlap between the ionization region and acceleration region within the SPT100 discharge chamber: ions are created throughout a region in space over which the potential varies greatly, thus the spread in the I(V) curve reflects the fact that ions are "born" in regions of different potential and therefore experience different acceleration voltages. This spread is in no way equal to the traditional thermal variation in ion energy. The definition of ion temperature to be used in this report is therefore strictly mathematical and in no way implies any form of thermal equilibrium.

The ion temperature will be defined analogous to that of a traditional Maxwellian thermal distribution. The one-dimensional Maxwellian distribution written in terms of the peculiar velocity, $\mathrm{u}_{\mathrm{i}}-\mathrm{u}_{\mathrm{d}}$ :

Eqn. 11

$$
f\left(u_{i}\right)=\sqrt{\frac{\beta}{\pi}} \exp \left(-\beta\left(u_{i}-u_{d}\right)^{2}\right) .
$$

written in terms of energy the peculiar velocity is

Eqn. 12

$$
u_{i}-u_{d}=\left(\frac{2 \sqrt{\left(E_{i}-E_{d}\right)^{2}}}{m_{i}}\right)^{\frac{1}{2}}
$$

so that now

Eqn. 13

$$
\mathrm{f}\left(\mathrm{E}_{\mathrm{i}}\right)=\sqrt{\frac{\beta}{\pi}} \exp \left(\frac{-2 \beta}{\mathrm{m}_{\mathrm{i}}} \sqrt{\left(\mathrm{E}_{\mathrm{i}}-\mathrm{E}_{\mathrm{d}}\right)^{2}}\right) .
$$

Substituting for $\beta=\mathrm{m}_{\mathrm{i}} / 2 \mathrm{kT}$ and recognizing that $k \mathrm{~T}_{\mathrm{i}} / \mathrm{e}$ is the equivalent temperature in electron-volts, $T_{e v}$, yields the final result

Eqn. 14



According to Eqn. 14 for a Maxwellian energy distribution, the ion temperature in $\mathrm{eV}$ represents the half-width of $\mathrm{f}\left(\mathrm{E}_{\mathrm{i}}\right)$ at the point where $\mathrm{f}\left(\mathrm{E}_{\mathrm{i}}\right)$ has a value of $e^{-1}$ times the peak value (where $E_{i}=E_{d}$ at the peak). Mathematically, 
Eqn. 15

$$
\frac{\left(\left(E_{i}-E_{d}\right) / q e\right.}{T_{e v}}=1
$$

where $f\left(E_{i}\right)=0.37 f\left(E_{d}\right)$.

In the context of the Maxwellian analysis, the temperature of the I(V) distributions obtained with the 45-degree analyzer will be defined as the half width of the I(V) distribution at the point in the curve where $I(V)$ is equal to 0.37 times $I\left(V_{m}\right)$, where $V_{m}$ represents the most probable voltage such that $I\left(V_{m}\right)$ is a maximum. This value of ion "temperature" will be denoted as $\tau_{\mathrm{i}}$, but, as stated earlier, usage of the term does not imply any type of equilibrium. Instead, $\tau_{\mathrm{i}}$ simply reflects an indication of the width-to-height aspect ratio of the $I(V)$ distribution.

With this in mind trends in the extensive $I(V)$ data sets were evaluated. In order to define a unique temperature the definition of $\tau_{i}$ requires the $I(V)$ distribution to be symmetric about the drift energy, $E_{d}$. Since the curves obtained in this research were nonsymmetric the definition of $\tau_{\mathrm{i}}$ implied two distinct temperatures corresponding to the $0.37 \times f\left(E_{d}\right)$ point on either side of the peak. For discussion purposes the temperature was defined as the $0.37 \times f\left(E_{d}\right)$ point such that $E>E_{d}$ (to the right of the peak).

The 0.5-m I(V) data sets exhibited interesting general trends in ion temperature. The interpretation of $\tau_{\mathrm{i}}$ was somewhat confused by the existence of multiple peaks for the angular positions centered around 90 and -90 degrees; for these traces it was unclear which peak defined the main distribution. However, for many of the angular positions the distribution was characterized by a single dominant peak which was used to calculate $\tau_{\mathrm{i}}$. Figure 25 shows a plot of $\tau_{\mathrm{i}}$ as a function of angular position for points lying $0.5 \mathrm{~m}$ away from the SPT-100 exit plane. Values of $\tau_{\mathrm{i}}$ were not calculated for points in which the choice of dominant distribution peak was unclear.

Variation in the ion temperature for points lying along the $1.0 \mathrm{~m}$ radius was also analyzed. However, the anomalous distributions found between 5 degrees and 20 degrees of thrust axis prevented calculation of a meaningful $\tau_{i}$ for these points: the distribution in this regime consisted of multiple current peaks with comparable magnitudes such that the choice of a dominant distribution was not clear. This fact created "gaps" in the $\tau_{\mathrm{i}}$ vs $\theta$ plot shown as Figure 26 and hindered the identification of trends.

The calculated values for $\tau_{\mathrm{i}}$ shown in Figure 25 and Figure 26 demonstrate excellent agreement with the currently accepted structure of the ionization and acceleration regions within the SPT-100 discharge chamber. Baranov, et al. ${ }^{11}$ have recently developed a comprehensive model of the acceleration layer formation within the Hall thruster. This model was used to predict plasma parameters such as electron temperature, plasma density, collision frequencies, and electric field within the acceleration region of a Hall thruster very similar to the SPT-100. The neutral atom density and plasma potential predictions resulting from this model are reproduced as Figure 27. 
In this model, as in actual Hall thruster operation, neutral propellant is injected through the anode. This propellant is then ionized by electron collisions as the neutral atoms travel towards the cathode. The decay in neutral atom density with distance from the anode therefore corresponds to the disappearance of neutrals due to ionization. As can be seen from the model, most of the propellant is ionized within a region extending $2.5 \mathrm{~cm}$ from the anode. Within this ionization region the local plasma potential varies from 300 to $250 \mathrm{~V}$; thus the ions that are "born" within this region will have a voltage spread of approximately $50 \mathrm{~V}$ upon exiting the discharge chamber. This agrees well with the measured values of $\tau_{i}$ (half-width) of approximately 20 to $40 \mathrm{~V}$ for the main discharge ion beam within 90 degrees of thrust axis shown in Figure 25 and Figure 26. Furthermore, many of the ions formed within the first $2.5 \mathrm{~cm}$ of the anode will suffer a neutralizing collision with the discharge chamber wall downstream, followed by a second (or even third) ionizing electron collision; these ions will increase the spread in the exhaust voltage distribution beyond that induced by the $2.5 \mathrm{~cm}$ ionization zone.

The population of ions behind the thruster (at angles greater than about 100 degrees) posses considerably lower temperature than the main beam ions as expected. However, this backflow plasma still has a value of $\tau_{\mathrm{i}}$ ranging between 2 and $5 \mathrm{~V}$. Although no investigations of the Hall thruster backflow regions were performed prior to this study, it was widely accepted that this region most likely consisted of macroscopically stagnant plasma arising from charge-exchange collisions between plume ions and background facility gas due to vacuum chamber pumping limitations; thus the distribution would have a width on the order of the local ambient neutral temperature $(300 \mathrm{~K}$, or about $0.03 \mathrm{eV})$. However, it is very unlikely that the high temperature implied by the width of the measured voltage distribution in the backflow (of roughly 58,000 K) represents a true thermal spread within a macroscopically stagnant plasma as would be expected to exist behind the thruster. The physical mechanism producing such a wide energy spread in the backflow ions is unknown as of this writing. Further characterization of the backflow region is thus necessary.

\subsection{Most Probable Voltage}

As another measure of the ion energy structure the most probable ion voltage was compiled as a function of angular position. This voltage was easily defined and identifiable on all plots as the voltage (energy/q) corresponding to the maximum in the I(V) vs V curve. This quantity is plotted for both $0.5 \mathrm{~m}$ and $1.0 \mathrm{~m}$ in Figure 28 . The angular energy structure displayed in Figure 28 exhibits some intriguing qualities, the most striking of which is the existence of high-energy ions at angles exceeding 90 degrees from the thrust axis: ions with $\mathrm{V}_{\mathrm{m}}$ on the order of $250 \mathrm{~V}$ persist out to 100 degrees, while particles with $\mathrm{V}_{\mathrm{m}}$ nearly $100 \mathrm{~V}$ extend to nearly 130 degrees at $0.5 \mathrm{~m}$. Although the $1.0 \mathrm{~m}$ data show high energy ions up to $100 \mathrm{~V}$ at angles of 100 degrees, the extent of the $250 \mathrm{~V}$ ions is narrower at $1.0 \mathrm{~m}$ then at $0.5 \mathrm{~m}$, with these high-energy ions decreasing at 70 to 80 degrees off axis. In general, the trends in angular evolution of most probable ion energy at $1.0 \mathrm{~m}$ appears to be a "pinched" version of that at $0.5 \mathrm{~m}$. This pinching effect may be due to the configuration of the plasma electric field within the plume. A discussion of this effect can be found in Reference 12.

Although the plasma electric field may explain the narrowing of the highly divergent ions between $0.5 \mathrm{~m}$ and $1.0 \mathrm{~m}$, the existence of such high-energy ions at angles exceeding 90 degrees off axis is still puzzling. Ions with acceleration voltages on the order of the discharge voltage of $300 \mathrm{~V}$ must be formed near the upstream end of the thruster 
acceleration layer and, thus, well upstream of the thruster exit plane. In order for these ions to be emitted at angles near and exceeding 90 degrees off axis they would have to undergo a considerable curvature in their trajectory upon exiting the thruster since there is no direct line-of-sight from these points to the inside of the discharge chamber.

Within this picture of the acceleration layer formation it is apparent that a significant force would be required to deflect high-energy ions originating from deep in the discharge chamber out to trajectories approaching and exceeding 90 degrees off axis. The existence of such a force is improbable. It is more plausible that a small amount of propellant ionization and radial acceleration occurs downstream of the discharge chamber exit, external to the thruster. Since the electron mobility is very large along magnetic field lines within the discharge chamber the magnetic field lines represent electric equipotentials. Thus ions are formed and accelerated into trajectories normal to the magnetic field line at the ion formation point. Bending of the magnetic field lines outward from the exit plane would produce a magnetic field fringe with a normal approaching 90 degrees. Ions formed within this downstream region would experience an acceleration force perpendicular to the thrust vector and would therefore appear at large angles. However, although conceptually this scenario seems plausible, previous modeling and experimental probing of the near-exitplane region in Hall thrusters has not documented electric and magnetic fields downstream of the discharge chamber of sufficient strength to produce ions with radial voltages on the order of the applied discharge voltage. It is clearly apparent that this phenomenon is poorly understood and requires further investigation.

\subsection{Multiple Peak Structure}

Possibly the most striking feature uncovered in the analysis of the ion energy distribution function as approximated by $I(V)$ curves was the existence of multiple current peaks suggesting discrete distributions for many angular positions. As can be seen from examining these structures these peaks always occurred at discrete multiples of voltage, e.g. a primary peak at $\mathrm{V}_{b}$, with secondary peaks at $\mathrm{V}_{\mathrm{b}} / 3, \mathrm{~V}_{\mathrm{b}} / 2,3 \mathrm{~V}_{\mathrm{b}} / 2,2 \mathrm{~V}_{\mathrm{b}}$, and $3 \mathrm{~V}_{\mathrm{b}}$. The explanation for this structure is based on the hypothesis of charge-exchange collisions occurring within the plume plasma.

Consider a charge-exchange collision between an ion with charge $q=2$ accelerated through a voltage of $\mathrm{V}_{\mathrm{b}}$ (having energy $E=q \mathrm{~V}_{b}$ ) and a slow neutral atom. The definition of a charge-exchange collision is an interaction during which one or more electrons are transferred with no significant transfer of kinetic energy between the reactants. Therefore the transfer of an electron from the neurral to the $q=1$ reactant will produce a slow product ion with charge $q=1$ along with an ion with kinetic energy still equal to $E$, but with charge reduced from $\mathrm{q}=2$ to $\mathrm{q}=1$. Thus the fast product ion will have an equivalent voltage (energy-per-charge) of $2 V_{b}$. Such a scenario can be easily envisioned for two ionic reactants as well: a $q=1$ ion and $\mathrm{q} q=2$ ion, both accelerated through a voltage of $\mathrm{V}_{b}$, can

produce product ions with equivalent voltages of $2 \mathrm{~V}_{b}$ and $\mathrm{V}_{b} / 2$. All possible products of such reactions relevant to the Hall thruster plume are represented in Table 2 and Table 3. 
Reactants at $\mathrm{V}_{\mathrm{b}}$

Ion Products

Electrons transferred

\begin{tabular}{|c|c|c|}
\hline$X \mathrm{e}^{2+}+\mathrm{Xe}^{+}$ & $\mathrm{Xe}^{+}=2 \mathrm{~V}_{\mathrm{b}}$ with $\mathrm{Xe}^{2+}=\mathrm{V}_{\mathrm{b}} / 2$ & 1 \\
\hline $\mathrm{Xe}^{2+}+\mathrm{Xe}^{+}$ & $X e^{3+}=2 V_{b} / 3$ & 1 \\
\hline $\mathrm{Xe}^{2+}+\mathrm{Xe}^{+}$ & $\mathrm{Xe}^{3+}=\mathrm{V}_{\mathrm{b}} / 3$ & 2 \\
\hline $\mathrm{Xe}^{3+}+\mathrm{Xe}^{+}$ & $\mathrm{Xe}^{2+}=3 \mathrm{~V}_{\mathrm{b}} / 2$ with $\mathrm{Xe}^{2+}=\mathrm{V}_{\mathrm{b}} / 2$ & 1 \\
\hline $\mathrm{Xe}^{3+}+\mathrm{Xe}^{+}$ & $X e^{4+}=3 V_{b} / 4$ & 1 \\
\hline $\mathrm{Xe}^{3+}+\mathrm{Xe}^{+}$ & $\mathrm{Xe}^{+}=3$ with $\mathrm{Xe}^{3+}=\mathrm{V}_{\mathrm{b}} / 3$ & 2 \\
\hline $\mathrm{Xe}^{3+}+\mathrm{Xe}^{+}$ & $\mathrm{Xe}^{4+}=\mathrm{V}_{\mathrm{b}} / 4$ & 3 \\
\hline $\mathrm{Xe}^{3+}+\mathrm{Xe}^{2+}$ & $X e^{2+}=3 V_{b} / 2$ with $X e^{3+}=2 V_{b} / 3$ & 1 \\
\hline $\mathrm{Xe}^{3+}+\mathrm{Xe}^{2+}$ & $\mathrm{Xe}^{4+}=3 \mathrm{~V}_{\mathrm{b}} / 4$ with $\mathrm{Xe}^{+}=2 \mathrm{~V}_{\mathrm{b}}$ & 1 \\
\hline$X e^{3+}+X e^{2+}$ & $\mathrm{Xe}^{+}=3 \mathrm{~V}_{\mathrm{b}}$, with $X \mathrm{e}^{4+}=\mathrm{V}_{\mathrm{b}} / 2$ & 2 \\
\hline
\end{tabular}

Table 2. Possible reactant and product combinations for CE collisions occurring between two high-energy beam ions, each with voltage $V_{b}$.

\begin{tabular}{|c|c|c|}
$\begin{array}{c}\text { Reactant ion at } \mathbf{V}_{\mathrm{b}} \\
\text { with } \mathbf{0} \mathbf{V} \text { neutral }\end{array}$ & $\begin{array}{c}\text { Ion products with } \\
\text { Energy }>\mathbf{0}\end{array}$ & Electrons Transferred \\
\hline $\mathrm{Xe}^{+}+\mathrm{Xe}$ & none & N/A \\
\hline $\mathrm{Xe}^{2+}+\mathrm{Xe}$ & $\mathrm{Xe}^{+}=2 \mathrm{~V}_{\mathrm{b}}$ & 1 \\
\hline $\mathrm{Xe}^{3+}+\mathrm{Xe}$ & $\mathrm{Xe}^{2+}=3 \mathrm{~V}_{\mathrm{b}} / 2$ & 2 \\
\hline $\mathrm{Xe}^{3+}+\mathrm{Xe}$ & $\mathrm{Xe}^{+}=3 \mathrm{~V}_{\mathrm{b}}$ & 1 \\
\hline
\end{tabular}

Table 3. Possible reactant-product combinations for the CE collision between a beam ion with voltage $V_{b}$ and a stagnant background neutral.

As discussed in Reference 12, momentum transfer collisions are evidenced by monotonically decaying tails on the ion voltage distributions, while $\mathrm{CE}$ collisions are represented by "bump-on-tail" types of distributions. The measured ion voltage distributions near centerline at $0.5 \mathrm{~m}$ seem to be very consistent with a momentum-transfer elastic collision analysis between plume beam ions. A discussion of these momentum transfer collisions can be found in References 6 and 12. This paper concentrates on the appearance of CE collision signatures within the ion voltage distributions.

Many of the measured ion voltage distributions exhibit a "bump on tail" shape, where the voltage of the bump maximum is exactly twice the most probable voltage of the distribution. This can be seen, for example, by examining Figure 5: denoting $\mathrm{V}_{\mathrm{b}}$ as the most probable beam voltage, $V_{b}=235 \mathrm{~V}$ at 90 degrees with the bump occurring at $\mathrm{V}=470$ 
V. From Table 2 and Table 3 the possible reactions creating products with a voltage of twice the main beam voltage are (1) the CE collision between a $\mathrm{Xe}^{+}$beam ion with a $\mathrm{Xe}^{2+}$ beam ion (2) the CE collision between a $\mathrm{Xe}^{3+}$ beam ion and a $\mathrm{Xe}^{2+}$ beam ion, and (3) the $\mathrm{CE}$ collision between a $\mathrm{Xe}^{2+}$ beam ion with a background neutral. The distribution measured at 90 degrees will be used as a discussion tool to evaluate these collisions.

The second reaction mentioned above involved a collision between two minority species in the plasma. The probability of such a second-order collision is much less than that involving one or more majority species, such as $\mathrm{Xe}^{+}$or $\mathrm{Xe}$, therefore either the first or the third reaction seems most likely to be observed. If the reaction responsible for the peak at $2 \mathrm{~V}_{\mathrm{b}}$ in the 90-degree data is of the first type involving two beam ions, then a sibling bump must occur at a voltage of $\mathrm{V}_{\mathrm{b}} / 2=117 \mathrm{~V}$. The distribution does display a bump near $120 \mathrm{~V}$, however the width of this low-voltage bump is not supported by a CE analysis of the collision between two beam ions. Based on princples discussed at length in Reference 12 , assume that the large central peak in the 90 -degree data reflects the pre-collision distribution of $\mathrm{Xe}^{+}$and $\mathrm{Xe}^{2+}$ emitted from the thruster. A gaussian curve-fit to this portion of the curve is shown compared with the data at 90 degrees in Figure 29.

The CE reaction between these two pre-collision distributions of beam ions would then form a resultant post-collision distribution as shown compared with the data in Figure 31; in this figure the height of the post-collision distributions has been chosen arbitrarily to match the data. The post-collision distribution resulting from the CE collision results in a distribution with the same shape as the pre-collision population shifted and broadened by a factor of two coincidentally with a distribution shifted and narrowed by a factor of $1 / 2$. It is apparent from the figure that even though the shape of the high-voltage bump is well represented, the narrowed product distribution of doubly charged ions does not account for the breadth in the low-voltage portion of the data. Furthermore, arbitrarily choosing the peak height of the post-collision $\mathrm{Xe}^{2+}$ distribution to match the data implies that the doubly charged CE products comprise nearly $50 \%$ of the pre-collision ions and hence, that the precollision distribution of $\mathrm{Xe}^{2+}$ accounted for greater than $50 \%$ of the total flow.

It is apparent that a $\mathrm{CE}$ reaction between two pre-collision gaussian-like distributions of $\mathrm{Xe}^{+}$and $\mathrm{Xe}^{2+}$ cannot account for the measured shape of the voltage distribution function at 90 degrees. The other possible reaction producing a bump at $2 \mathrm{~V}_{b}$ is the collision between a $\mathrm{Xe}^{2+}$ beam ion and a background neutral. If the pre-collision distribution of $\mathrm{Xe}^{2+}$ was gaussian-like as discussed above, the beam ion/neutral collision would produce only a post-collision distribution centered at $2 \mathrm{~V}_{\mathrm{b}}$. This reaction does not explain the portion of the MBMS data below $200 \mathrm{~V}$ nor the portion between $300 \mathrm{~V}$ and 400 $\mathrm{V}$ (the resultant post-collision distribution would consist only of the central gaussian and the "bump" on the far right of Figure 31).

The trends of the MBMS data suggest another shape for the pre-collision distribution: the high-voltage tail above $300 \mathrm{~V}$ in the 90 -degree data has a nearly identical shape to the portion of the distribution less than $300 \mathrm{~V}$. With this in mind the following model is proposed. The portion of the 90-degree data curve to the left of $300 \mathrm{~V}$ represents the pre-collision distribution of singly and doubly charged xenon created and accelerated in the same region, while the high-voltage tail above $300 \mathrm{~V}$ represents $\mathrm{Xe}^{2+}$ beam ions which have gained an electron through a CE collision with a background neutral. The precollision $\mathrm{Xe}^{2+}$ distribution is thus calculated by multiplying the portion of the MBMS data between 0 and $300 \mathrm{~V}$ by some fraction. This is shown graphically in Figure 32.

A CE collision between the doubly charged xenon and background neutral would then produce a distribution of singly charged product ions with the same shape as the precollision distribution shifted and broadened by a factor of 2 . Figure 33 shows the postcollision distribution formed through such a shape-preserving reaction compared with the 
data. In this plot the height of the post-collision distribution has been chosen to match the data, but the distribution shape was determined by "cutting and pasting" the original data and is thus an exact duplicate of the portion of the curve between 0 and $300 \mathrm{~V}$.

It is apparent from an examination of Figure 33 that the high-voltage tail in the 90 degree ion voltage distribution is exactly the same as the shape of the curve between 0 and $300 \mathrm{~V}$; such a distribution can be explained through the occurrence of CE collisions between doubly charged xenon ions and ambient background neutrals. This result makes physical sense: the original pre-collision distribution of ions produced in the Hall thruster goes to zero at $300 \mathrm{~V}$ and thus does not contain any anomalous ions with voltages greater than that applied to the discharge. The high-voltage portion of the measured distribution function is the result of $\mathrm{CE}$ collisions occurring between the different ionic species within the plasma, namely between doubly charged ions and background neutrals.

Discussion of the collision signatures within the plume ion voltage distributions have been confined to the data recorded at $0.5 \mathrm{~m}$ radius from the SPT-100 up to this point. The data trends in the $1.0 \mathrm{~m}$ data exhibit many of the same tell-tale signatures of collisions, but the angular trends are much more intriguing. Excluding the anomalous zones at $1.0 \mathrm{~m}$ between 5 degrees and 20 degrees off axis the data display much of the same angular trends as the $0.5 \mathrm{~m}$ case. Referring to Figure 12 through Figure 18, the centerline voltage distribution exhibits somewhat of a monotonically decreasing tail associated with elastic collisions, with a CE-type signature superimposed. Ignoring, for now, the region between 5 and 20 degrees, the elastic-looking collision signatures evolve into typical CE structures very rapidly with increasing angle from centerline, with a "bump on tail" existing at precisely twice the most probable voltage of the main distribution. Furthermore, the height of these bumps is a larger fraction of the main discharge peak than exhibited in the $0.5 \mathrm{~m}$ data.

The voltage distributions measured between 5 degrees and 20 degrees, and -5 degrees and -20 degrees exhibit fascinating structure. These data were presented as Figure 19 through Figure 22. The voltage distributions of this region are characterized by extremely prominent $\mathrm{CE}$ peaks occurring at voltages greater than that which can be considered the "beam" voltage at approximately $260 \mathrm{~V}$. Referring to Table 3 it is apparent that this type of signature can arise from collisions between beam ions and background neutrals. Although there are possible reactions between beam ions that would produce peaks greater than $V_{b}$, as can be seen from Table 2 these reactions produce sibling products with energies less than $\mathrm{V}_{\mathrm{b}}$ simultaneously with the high-voltage peaks. The data do not exhibit any peaks with voltage less than $\mathrm{V}_{\mathrm{b}}$.

Between 5 and 6 degrees and between -5 and -7 degrees the "primary" distribution between 200 and $300 \mathrm{~V}$ associated with the thruster discharge acceleration process decays abruptly in magnitude. This decay in the $260 \mathrm{~V}$ peak is accompanied by a relative increase in the amplitude of the high-voltage peaks associated with CE collisions. The physics responsible for this process are straightforward. Consider a pre-collision distribution of singly, doubly, and triply charged ions that have been accelerated through the same voltage of $\mathrm{V}_{\mathrm{b}}$. If such a distribution were to pass through an ambient neutral background such that $100 \%$ of the ions experienced a CE collision with the background gas, the result would be all of the products listed in Table 3; 100\% of the reactants would become products and hence the "primary" pre-collision distribution with peak at $\mathrm{V}_{\mathrm{b}}$ would completely disappear, with only the product peaks at $3 \mathrm{~V}_{b} / 2,2 \mathrm{~V}_{b}$, and $3 \mathrm{~V}_{\mathrm{b}}$ present in the voltage distribution. If somewhat less of the ions experience a CE collision, say $50 \%$, the peak height of the $\mathrm{V}_{\mathrm{b}}$ distribution would be comparable with the high-voltage peaks since an equal number of pre-collision reactants and post-collision products exist. Thus, as the $\mathrm{V}_{\mathrm{b}}$ peak decays due to de-populating CE collisions with background gas the product peaks with voltage greater 
than $V_{b}$ will experience a simultaneous growth in amplitude. Furthermore, although the ionization fraction decreases with increasing ion charge state (e.g. there is less $\mathrm{Xe}^{3+}$ than $\mathrm{Xe}^{2+}$ ), the cross section for ion-neutral CE collisions scales according to $\mathrm{q}^{1.3}$ so that highly charged ions will demonstrate a disproportionate contribution to the CE signature. ${ }^{13,14}$

The data between 5 and 20 degrees off axis at $1.0 \mathrm{~m}$ display exactly such $\mathrm{CE}$ behavior. The data presented earlier is reproduced in part as Figure 34. The distribution peaks corresponding to the possible post-collision voltage multiples are clearly visible in the region between -7 and -11 degrees. The peak locations represent all possible products of the reactions considered previously in Table 3 along with the original population at $\mathrm{V}_{\mathrm{b}}=260 \mathrm{~V}$. Additionally, the width of the CE product distributions scale according to the voltage displacement from the main peak at $\mathrm{V}_{\mathrm{b}}$ : the peak at $2 \mathrm{~V}_{\mathrm{b}}$ is twice as wide as the primary distribution, the peak at $3 \mathrm{~V}_{\mathrm{b}}$ is three times as wide, etc. It is apparent, then, that the data at $1.0 \mathrm{~m}$ reflect extensive attenuation of the plume ion beam by $\mathrm{CE}$ collisions with background neutrals.

Subject to this interpretation, the ion voltage data between 5 and 20 degrees reveal a unique insight regarding the species distribution within the plasma. Consider the ion voltage trace taken at $1.0 \mathrm{~m}, 11$ degrees off axis shown in Figure 35 . In this trace a distribution peak with voltage equal to $4 \mathrm{~V}_{\mathrm{b}} / 3$ is clearly evident. The only possible mechanism to account for this peak requires the existence of a quadruply charged ion, i.e. $\mathrm{Xe}^{4+}$ exchanging a single electron with a background neutral. This result, then, implies that the electrons within the thruster discharge chamber are hot enough to produce $\mathrm{Xe}^{4+}$ ions.

The above discussion of charge-changing collisions was predicated on the assumption that the electron transfer was due to collisions between ions and neutrals. Identical behavior could also be explained by ion-electron recombination with free plasma electrons: if an ion were to recombine with an electron its charge state would be reduced and, hence, its energy/q increased. In consideration of this possibility a collisionalradiative examination of the free electron recombination within the plume plasma was conducted in Reference 12. The findings of this analysis showed that the time required for the plasma to decay to one-half its initial density was on the order of $200 \mathrm{sec}$. It is therefore apparent that ion-electron recombination is not significant over the short transit times required for an ion to travel a $1.0-\mathrm{m}$ path.

\section{Conclusions}

One of the most interesting contributions of this research was the measurement of the ion energy at angles exceeding 90 degrees off the thrust axis. Although of utmost importance to spacecraft integration, this low-density regime has historically been very difficult to probe. As can be seen by an examination of Figure $28, V_{m}$ is nearly $260 \mathrm{~V}$ for all positions within 100 degrees of the thrust axis at $0.5 \mathrm{~m}$ radius, with $\mathrm{V}_{\mathrm{m}}=90 \mathrm{~V}$ extending all the way around to -150 degrees at $1.0 \mathrm{~m}$ radius. The mechanism responsible for such high-energy ions extending into the backflow of the plume is not understood. It was previously believed that such high-energy ions must be formed deep within the thruster discharge chamber, and would therefore not have a direct line-of-sight path to the plume backflow. Since the existence of the force required to produce a trajectory with sufficient curvature to transport ions formed within the discharge chamber into the backflow is not justified, it is likely that these high-energy ions at large angles were formed downstream of the thruster exit plane and accelerated transverse to the thrust axis at voltages comparable to the applied voltage. These data suggest that the structure of the 
plasma acceleration region downstream of the thruster exit plane is not accurately explained by current models of Hall thruster operation.

A second remarkable finding regarding the ion energy distribution was the discovery of a bi-modal distribution existing in the region of 90 degrees off axis. Near thruster centerline the energy distribution was Gaussian-like with a most-probable voltage near the applied discharge voltage. With increasing angle off axis a secondary "hump" or plateau emerges on the low-energy side of the main peak. This evolution continues such that between 70 and 100 degrees and -70 and -110 degrees the ions posses two fairly distinct voltage distributions: the main distribution centered near the applied discharge voltage, and a secondary distribution of approximately one-half the magnitude of the main peak centered near one-half the applied voltage. With further increases in divergence angle this bi-modal distribution gives way rapidly to a stagnant background population. Although the secondary low-energy population seems to be centered very near $V_{m} / 2, a C E$ analysis of this phenomena does not seem to support the indicated width of the population. It is likely, then, that this distribution shape is a result of the thruster acceleration process and not a facility-induced error.

This research demonstrated direct documentation of facility perturbances on the Hall thruster plume structure. These parasitic effects were manifested by CE collisions between plume ions accelerated within the thruster and ambient background neutrals due to vacuum pumping limitations. The evidence for such ground-test facility errors was the existence of both reduced-charge ions and a corresponding flux of high-energy neutrals within 20 degrees of the thrust axis. As expected, these effects became more pronounced with increasing distance from the thruster.

The facility pressure during testing was approximately $3 \times 10^{-5}$ torr, representing a collision probability of $50 \%$ at $1.0 \mathrm{~m}$ from the thruster for the CE collision between $\mathrm{Xe}^{+}$ and background $\mathrm{Xe}$. If a facility with an order-of-magnitude improvement in pressure were utilized the collision probability at $1.0 \mathrm{~m}$ decreases to $6 \%$. It is apparent, then, that detailed plume characterization in the far-field of Hall thrusters (approaching and exceeding $1.0 \mathrm{~m}$ ) requiring high-accuracy should be performed at pressures on the order of $1 \times 10^{-6}$ torr or better to reduce parasitic signatures and more correctly quantify effects attributed to the thruster.

Some Hall thruster plume characterization will be insignificantly affected by these facility perturbations. For instance, measurements of plume sputtering on sample materials are fairly insensitive to CE collisions. Since the sputtering yield is dependent only on the incident atom energy (and not on the charge state), the charge-changing character of $\mathrm{CE}$ collisions will not affect the incident atom energy and the resultant sputtering rate will be unaffected.

\section{Acknowledgments}

This research benefited from the generous support of the Air Force Office of Scientific Research (AFOSR) represented by Dr. Mitat Birkan, the NASA-Lewis Research Center with equipment grants administered by Mr. John Sankovic, and support from the NASA-Johnson Space Center under the direction of Mr. Richard Barton. The unique opportunity to evaluate a state-of-the-art thruster was made available by a generous equipment loan from Mr. Mike Day of the Space Systems/Loral company. This support is gratefully acknowledged. Additionally, the authors would like to thank technicians Warren Eaton, Terry Larrow, Gary Gould, and Dave McLean for assitance with hardware 
fabrication. The first author would also like to thank the research staff of PEPL, namely, Colleen Marrese, Frank Gulczinski, James Haas, Sang-wook Kim, and George Williams for their discussions in the preparation of this manuscript. 


\section{References}

1 Absalamov, S., Andreev, V., Colbert, T., Day, M., Egorov, V., Gnizdor, R., Kaufman, H., Kim, V., Korakin, A., Kozubsky, K., Kudravzev, S., Lebedev, U., Popov, G., and Zhurin, V., "Measurement of plasma parameters in the Stationary Plasma Thruster (SPT-100) plume and its effect on spacecraft components," AIAA-92-3156, 28th AIAA / SAE / ASME / ASEE Joint Propulsion Conference, Nashville, TN, July 6-8, 1992.

2 Myers, R., and Manzella, D., "Stationary plasma thruster plume characteristics," IEPC-93-096, 23rd International Electric Propulsion Conference, Seattle, WA, Sept. 13-16, 1993.

Manzella, D., and Sankovic, J., "Hall thruster ion beam characterization," AIAA95-2927, 31st AIAA / ASME / SAE / ASEE Joint Propulsion Conference, San Diego, CA, July 10-12, 1995.

4 Pencil, E., "Preliminary far-field plume sputtering of the Stationary Plasma Thruster (SPT-100)," IEPC-93-098, 23rd International Electric Propulsion Conference, Seattle, WA, Sept. 13-16, 1993.

5 Pencil, E., Randolph, T., and Manzella, D., "End-of-life Stationary Plasma Thruster far-field plume characterization," AIAA-96-2709, 32nd AIAA / ASME / SAE / ASEE Joint Propulsion Conference, Lake Buena Vista, FL, July 1-3, 1996.

King, L.B., and Gallimore, A.D., "Propellant Ionization and Mass Spectral Measurements in the Plume of an SPT-100," AIAA-98-3657, 34th AIAA/ASME/SAE/ASEE Joint Propulsion Conference, Cleveland, Ohio, July 13$15,1998$.

7 deZeeuw, W., va der Ven, H., de Wit, J., and Donne, J., "An electrostatic time-offlight analyzer for simultaneous energy and mass determination of neutral particles," Rev. Sci. Instrum. 62 (1), Jan 1991, pp. 110-117.

8 Gaus, A, Htwe, W., Brand, T., and Schulz, M., "Energy spread and ion current measurements of several ion sources," Rev. Sci. Instrum. 65 (12), Dec. 1994, pp. 3739-3745.

Esaulov, V., Grizzi, O., Guillemot, L., Huels, M., Lacombe, S., and Vu Ngoc Tuan, "An apparatus for multiparametric studies of ion-surface collisions," Rev. Sci. Instrum. 50 (2), Feb. 1979, pp. 210-218.

King, L.B., and Gallimore, A.D, "Transport Property Measurements in the Plume of an SPT-100 Hall-effect Thruster," Journal of Propulsion and Power, Vol. 14, No. 3, May-June 1998, pp. 327-335.

11 Baranov, V., Nazarenko, Y., Petrosov, V., Vasin, A., and Yashnov, Y., "Energy model and mechanisms of acceleration layer formation for Hall thrusters," ALAA97-3047, 33rd AIAA/ASME/SAE/ASEE Joint Propulsion Conference, Viareggio, Italy, Oct. 14-17, 1991. 
King, L.B., Transport-property and Mass Spectral Measurements in the Plasma Exhaust Plume of a Hall-effect Space Propulsion System, Doctoral Dissertation, University of Michigan Dept. of Aerospace Engineering, Published through University Microfilms Internat'1, 1998.

13 Kusakabe, T., Horiuchi, T., Nagai, N., Hanaki, H., Konomi, I., and Sakisaka, M., "Charge transfer of multiply charged slow argon, krypton, and xenon ions on atomic and molecular targets. Single-charge transfer cross sections," Journal of Physics B: Atomic and Molecular Physics, Vol. 19, 1986, 2165-2174.

14 Koding, H., Pinkse, F., and Nibbering, N., "Double-electron transfer from Xe to $\mathrm{Xe}^{4+}$ at low energies as observed in the trap of a Fourier-transform ion cyclotron resonance mass spectrometer," Rapid Communications in Mass Spectrometry, Vol. 7, 1993, pp. 780-783. 


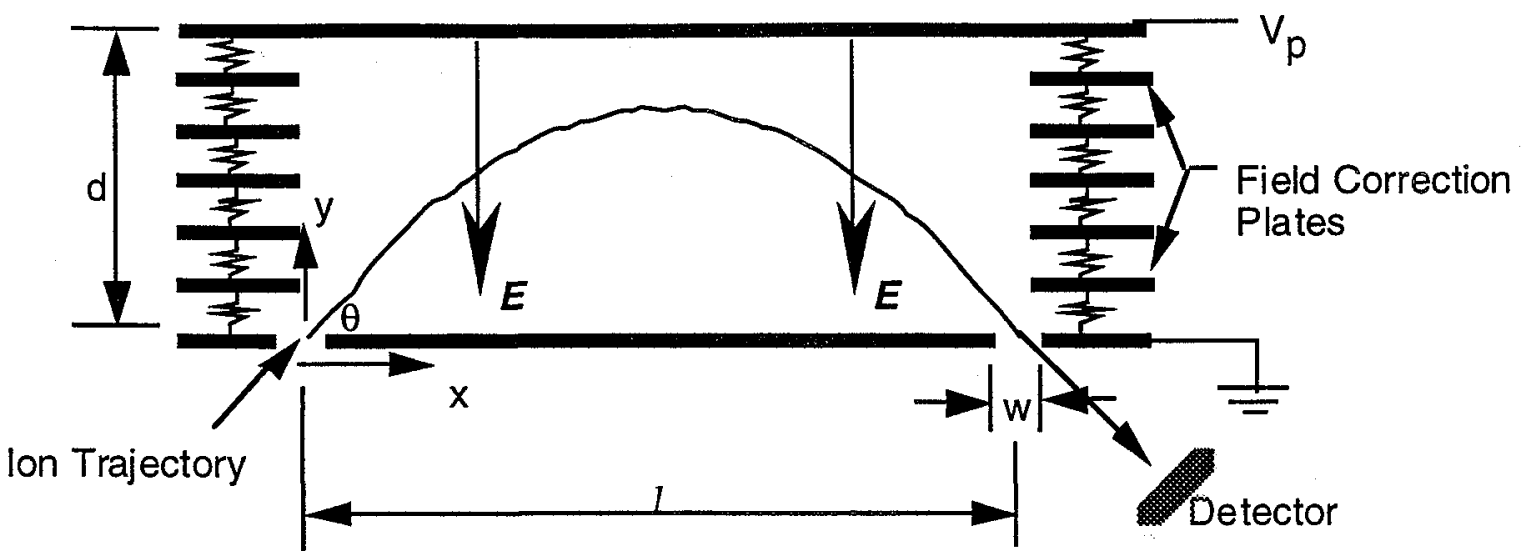

Figure 1. Schematic of 45-degree electrostatic ion energy analyzer. Constant electric field is formed by applying repelling voltage to top plate with bottom plate grounded. Field correction plates are biased with a voltage divider to force boundary conditions at mid-planes to prevent field distortion due to surrounding ground potential.
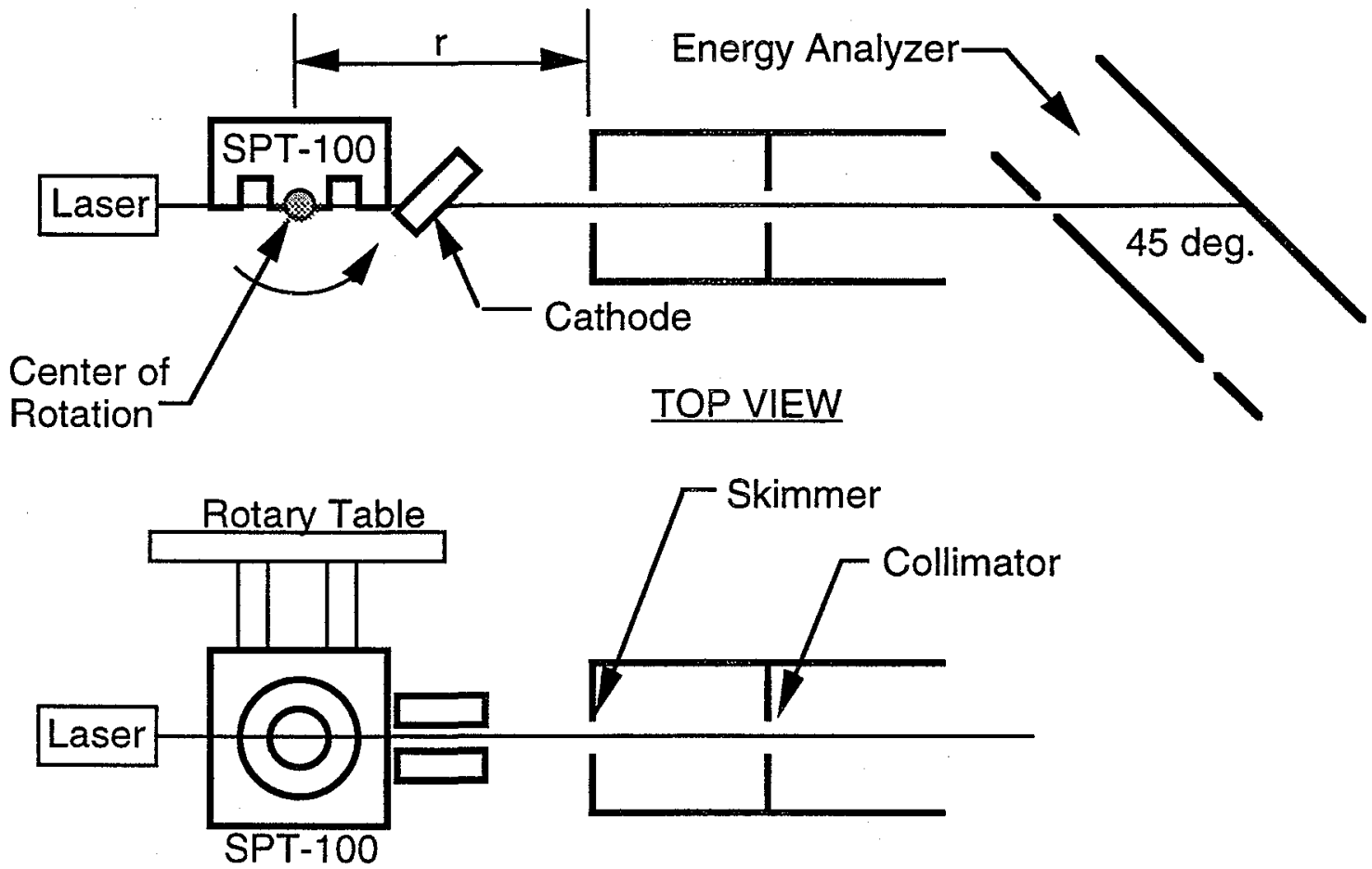

\section{SIDE VIEW}

Figure 2. Experimental set-up diagram showing rotary thruster mount and laser alignment of beam line. 


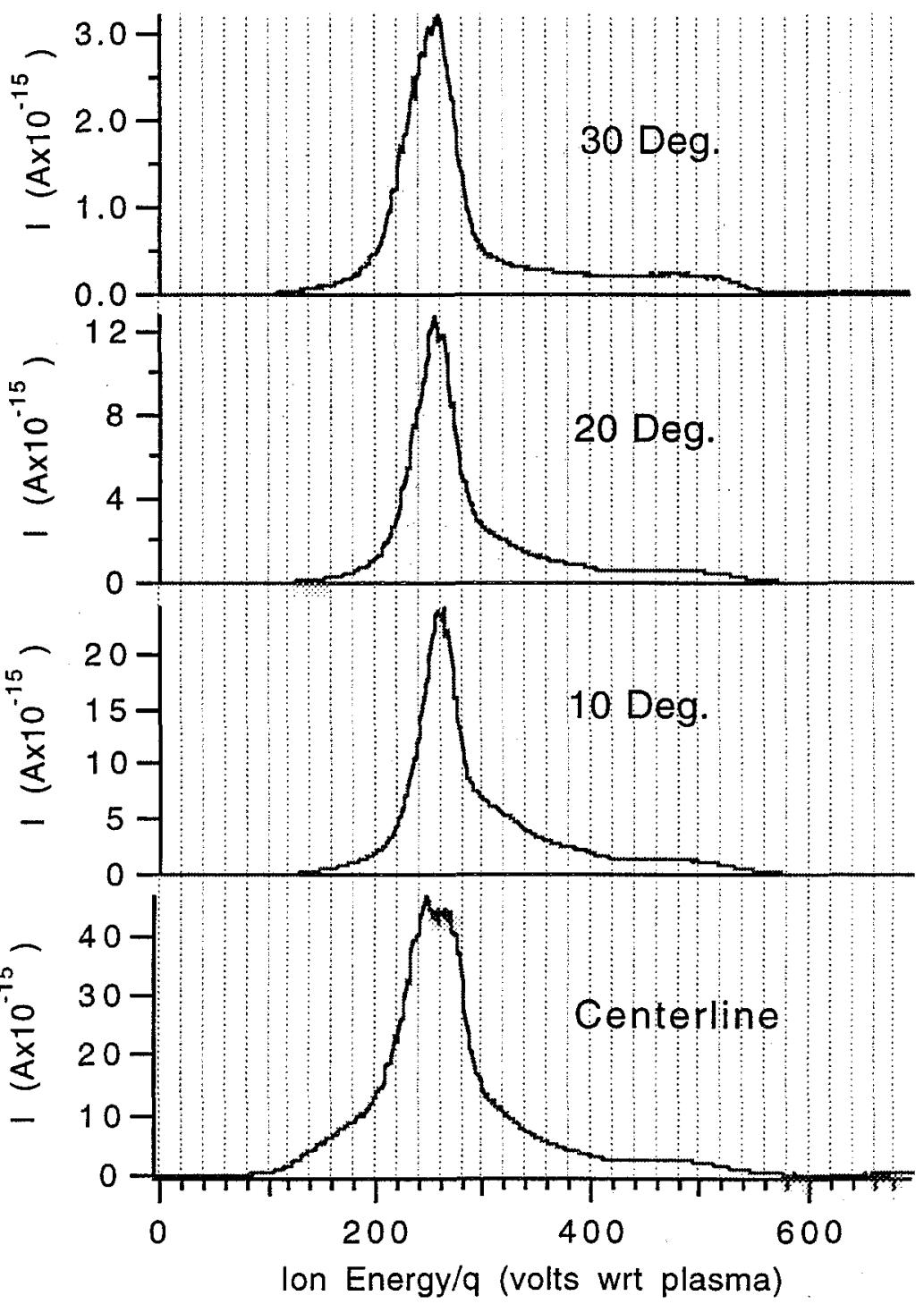

Figure 3. Ion current as a function of ion voltage at $0.5 \mathrm{~m}$ radius from the SPT-100 along the thrust axis and for points at 10, 20, and 30 degrees off axis. 


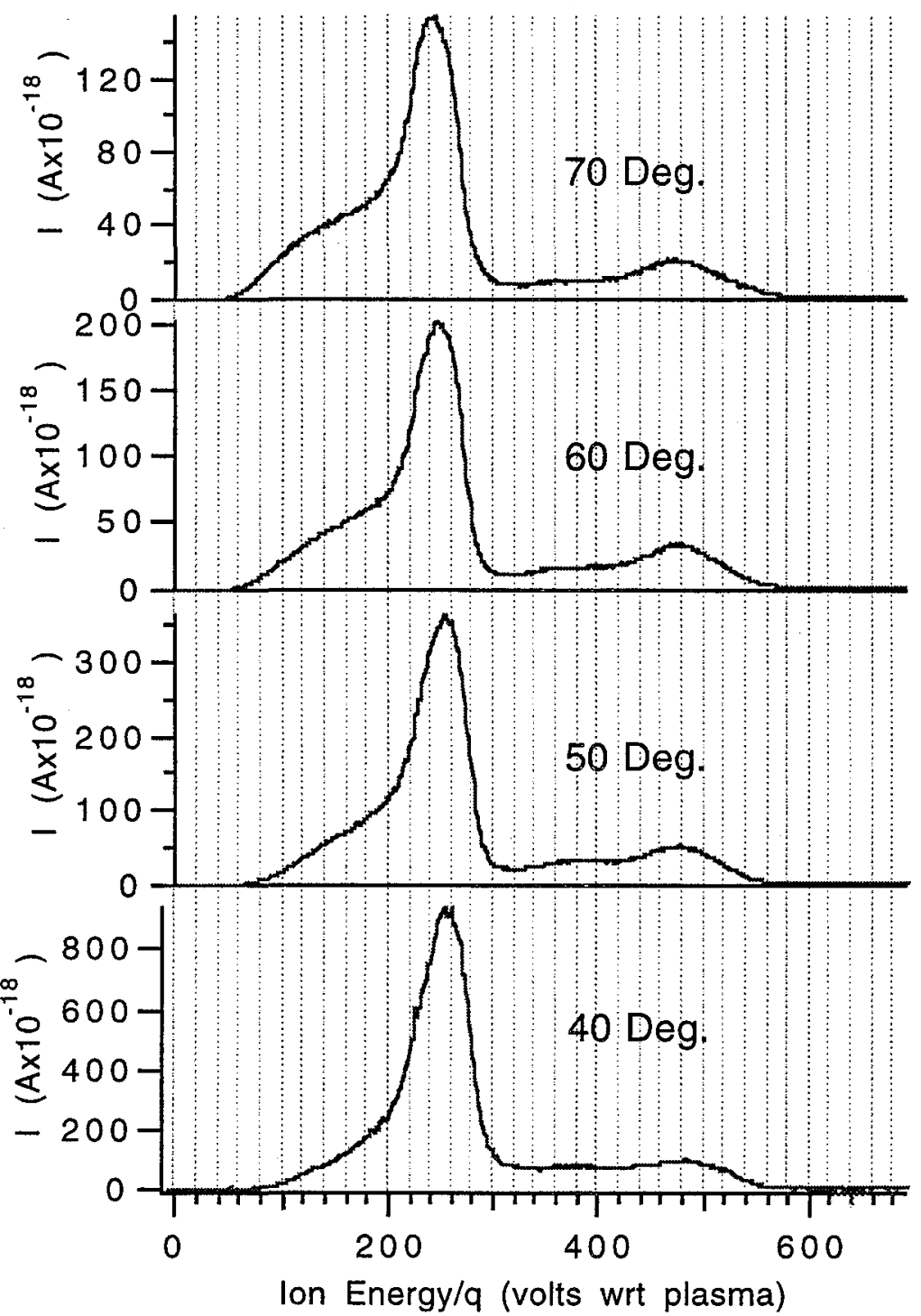

Figure 4. Ion current as a function of ion voltage at $0.5 \mathrm{~m}$ radius from the SPT-100 for points at 40 , 50,60 , and 70 degrees off thrust axis. 


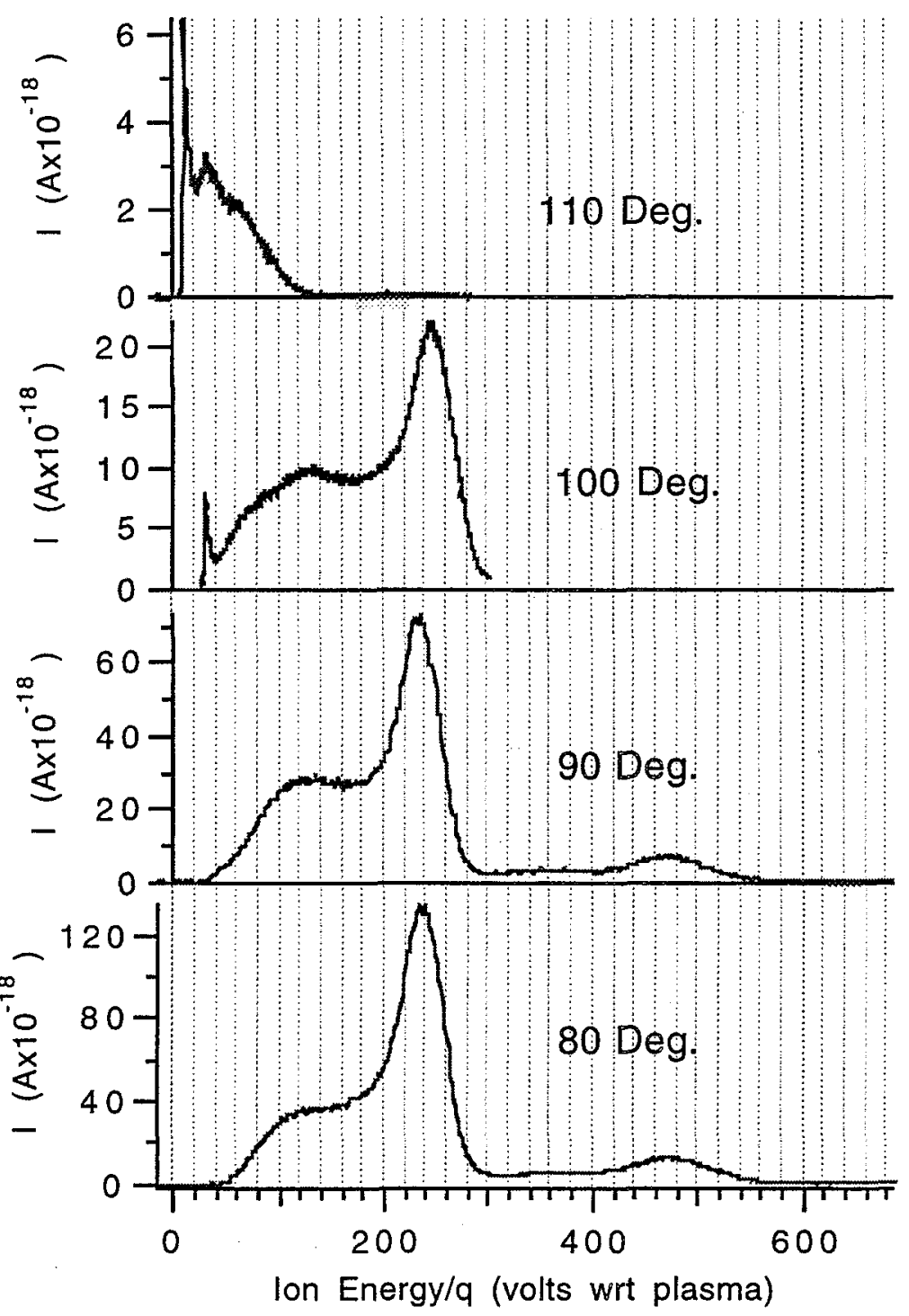

Figure 5. Ion current as a function of ion voltage at $0.5 \mathrm{~m}$ radius from the SPT-100 for points at 80 , 90,100 , and 110 degrees off thrust axis. 

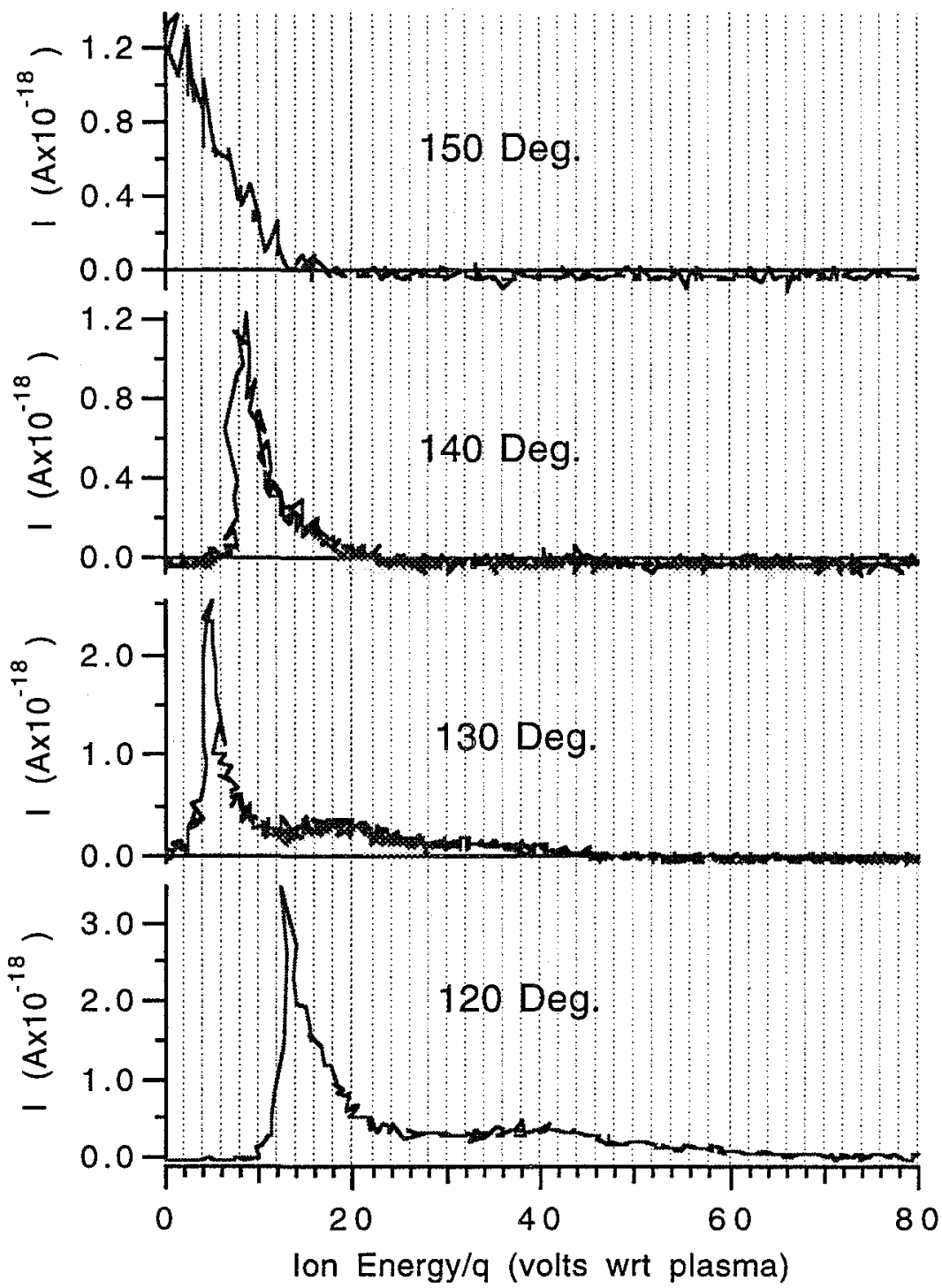

Figure 6. Ion current as a function of ion voltage at $0.5 \mathrm{~m}$ radius from the SPT-100 for points at $120,130,140$, and 150 degrees off thrust axis. 

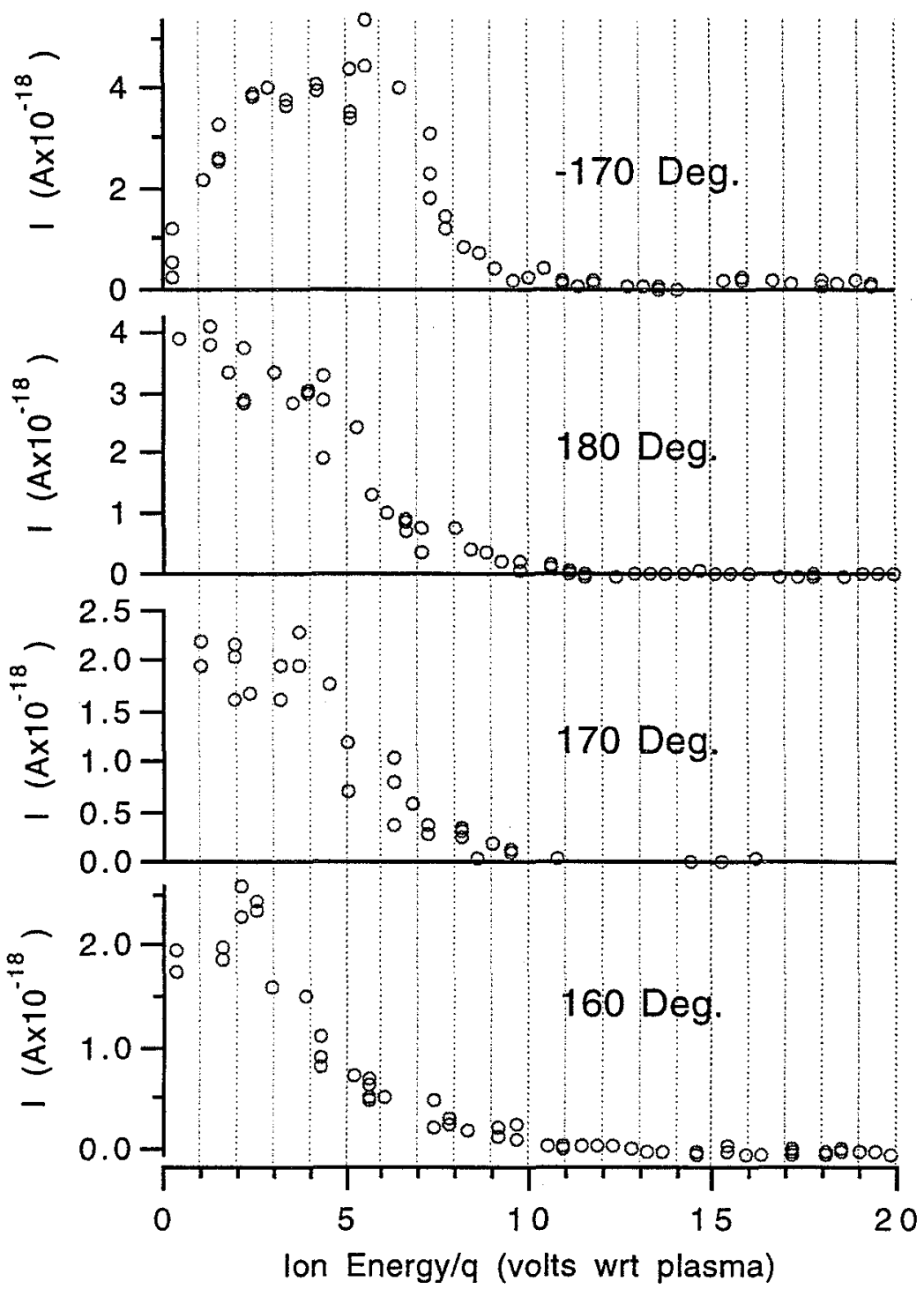

Figure 7. Ion current as a function of ion voltage at $0.5 \mathrm{~m}$ radius from the SPT-100 for points at $160,170,180$, and -170 degrees off thrust axis. 

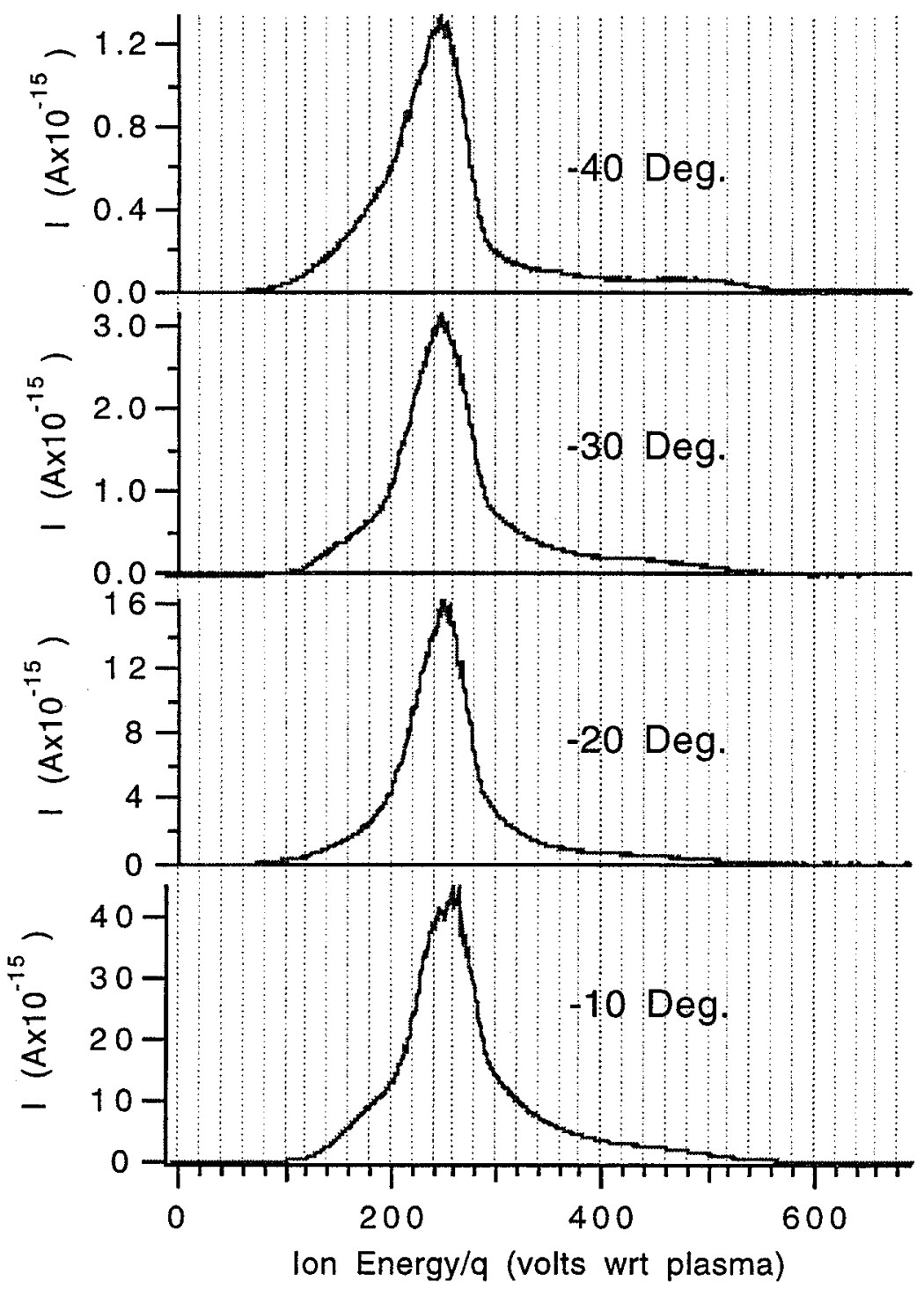

Figure 8. Ion current as a function of ion voltage at $0.5 \mathrm{~m}$ radius from the SPT-100 for points at $10,-20,-30$, and -40 degrees off thrust axis. 


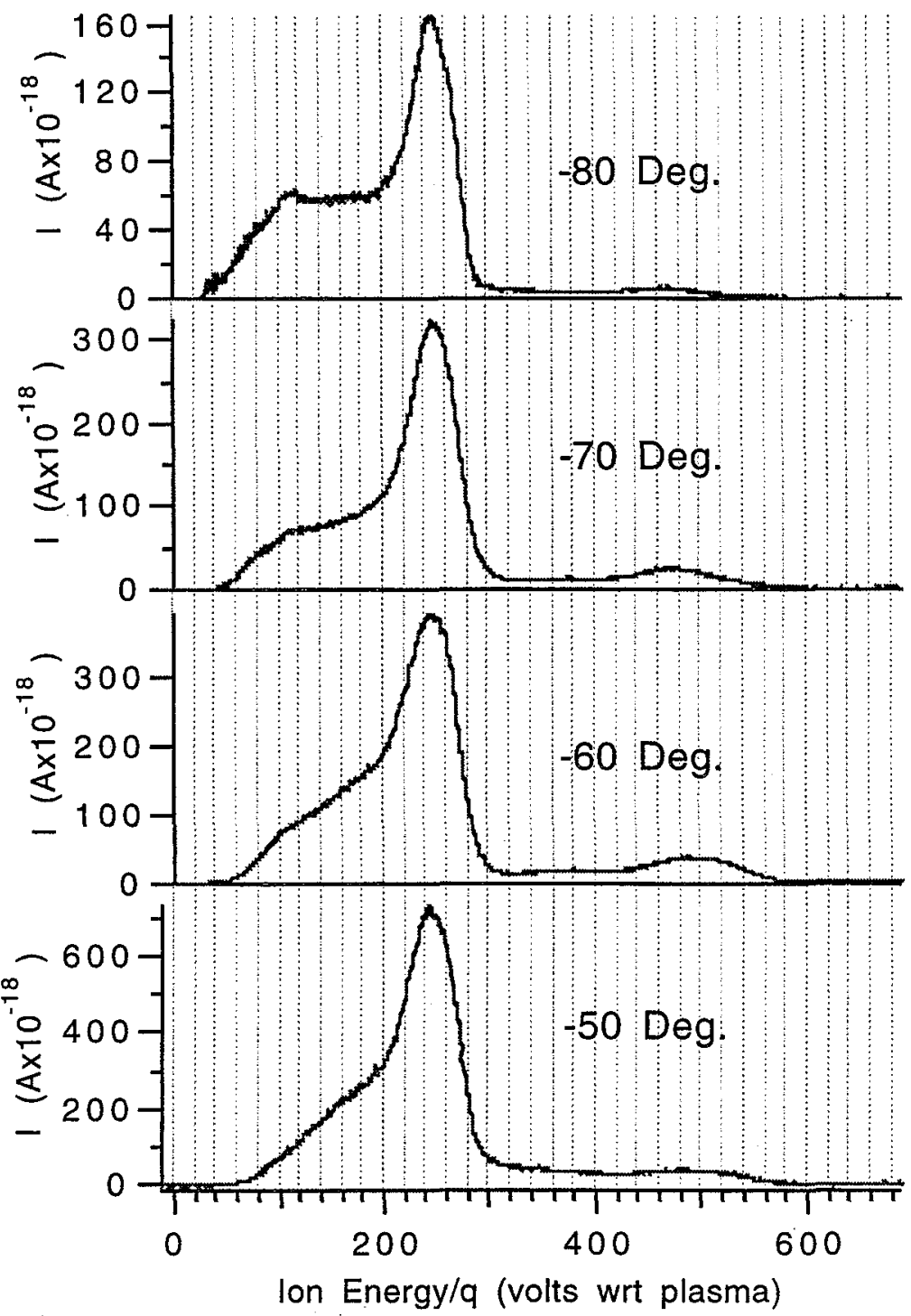

Figure 9. Ion current as a function of ion voltage at $0.5 \mathrm{~m}$ radius from the SPT-100 for points at $50,-60,-70$, and -80 degrees off thrust axis. 

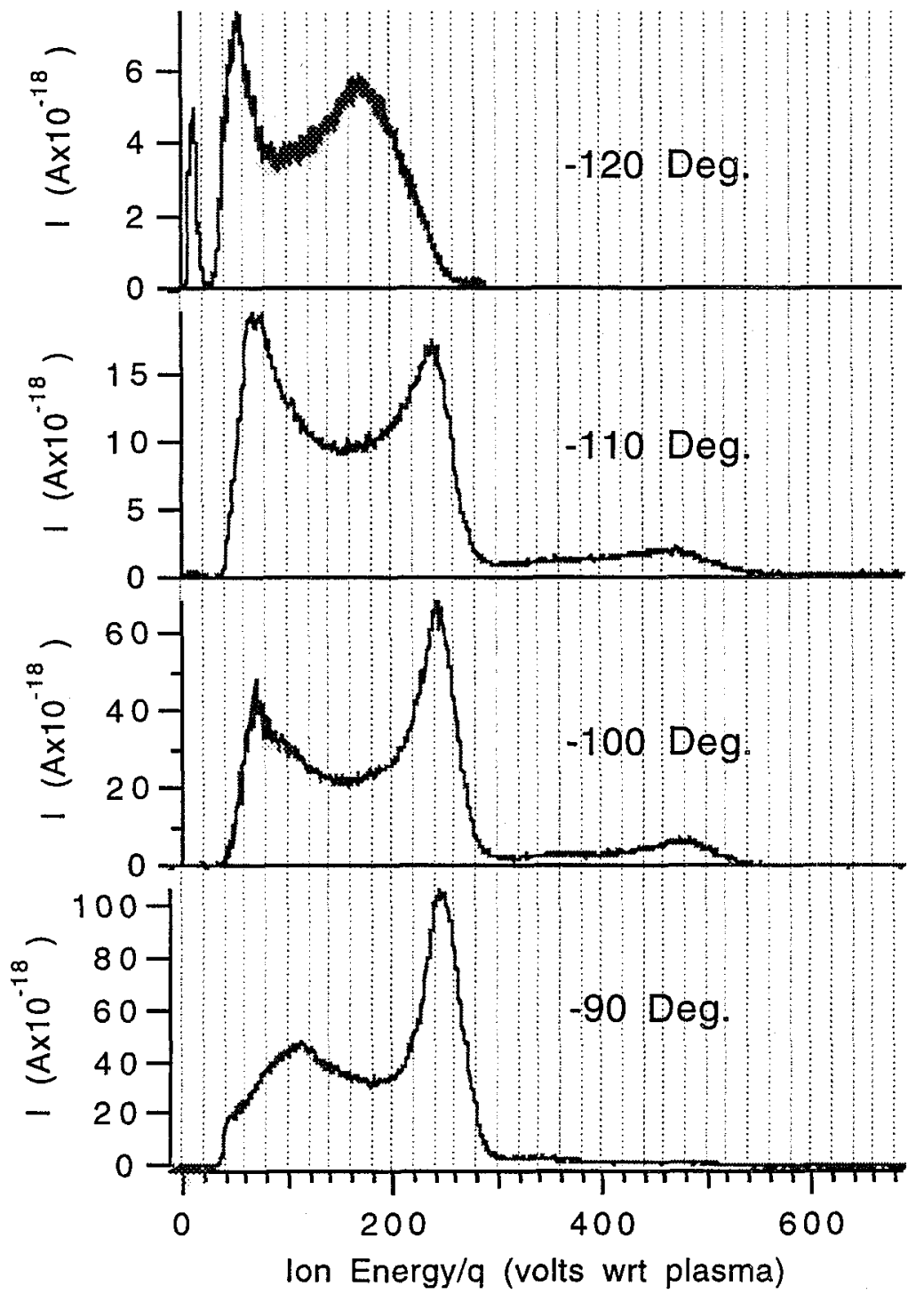

Figure 10. Ion current as a function of ion voltage at $0.5 \mathrm{~m}$ radius from the SPT-100 for points at $90,-100,-110$, and -120 degrees off thrust axis. 

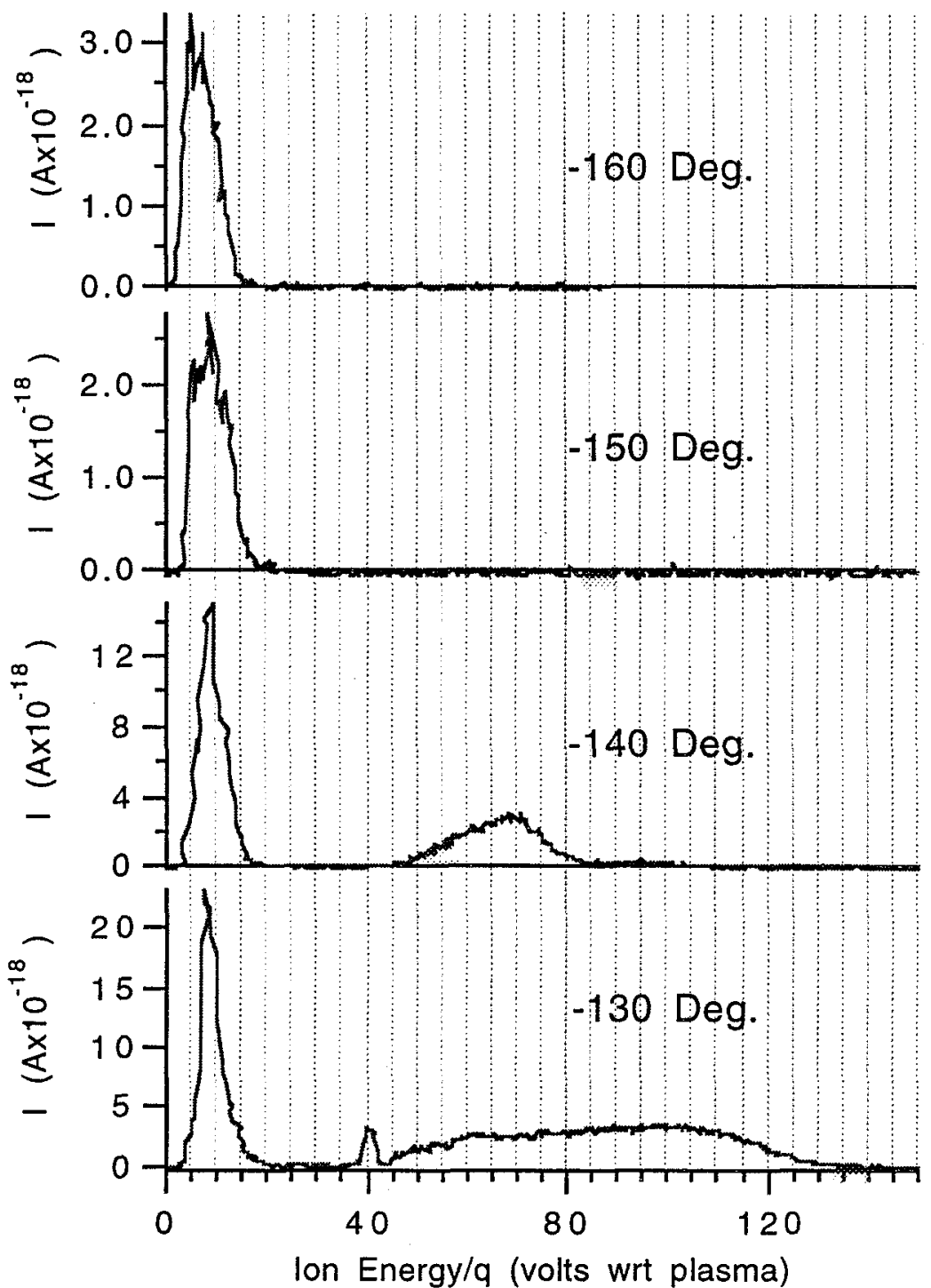

Figure 11. Ion current as a function of ion voltage at $0.5 \mathrm{~m}$ radius from the SPT-100 for points at $130,-140,-150$, and -160 degrees off thrust axis. 


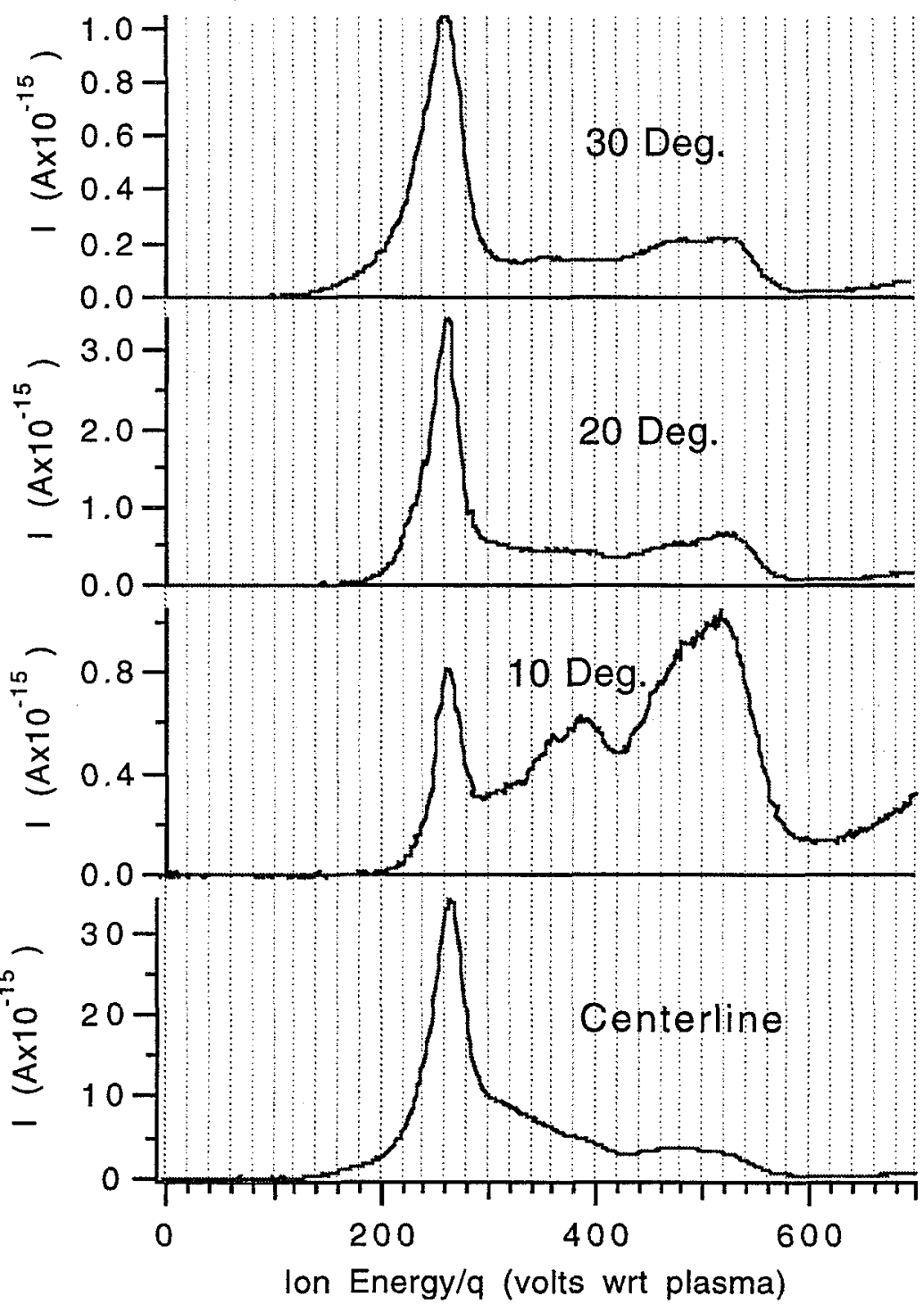

Figure 12. Ion current as a function of ion voltage at $1.0 \mathrm{~m}$ radius from the SPT-100 on the thrust axis in addition to points at 10, 20, and 30 degrees off axis. 


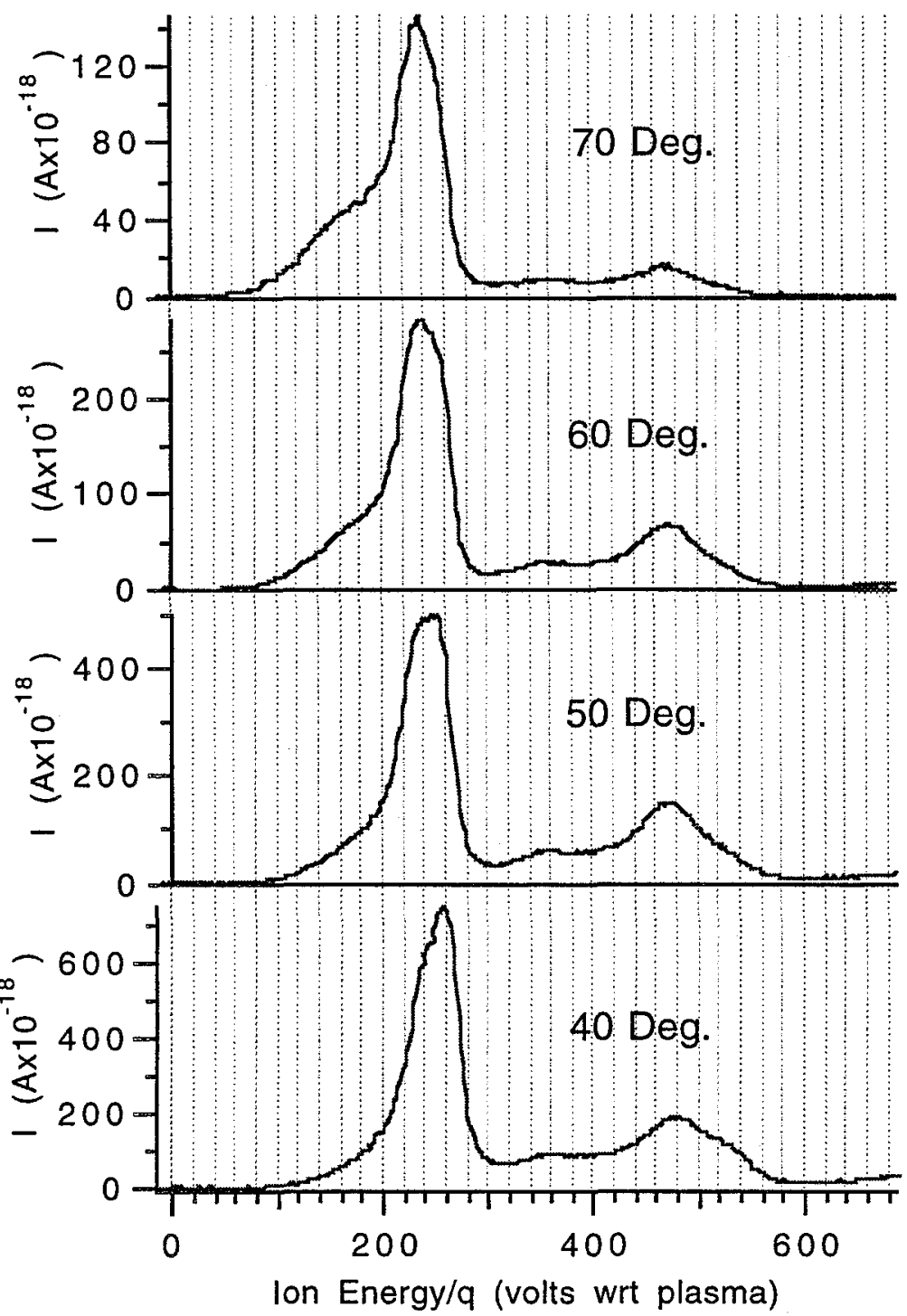

Figure 13. Ion current as a function of ion voltage at $1.0 \mathrm{~m}$ radius from the SPT-100 for points at $40,50,60$, and 70 degrees off thrust axis. 


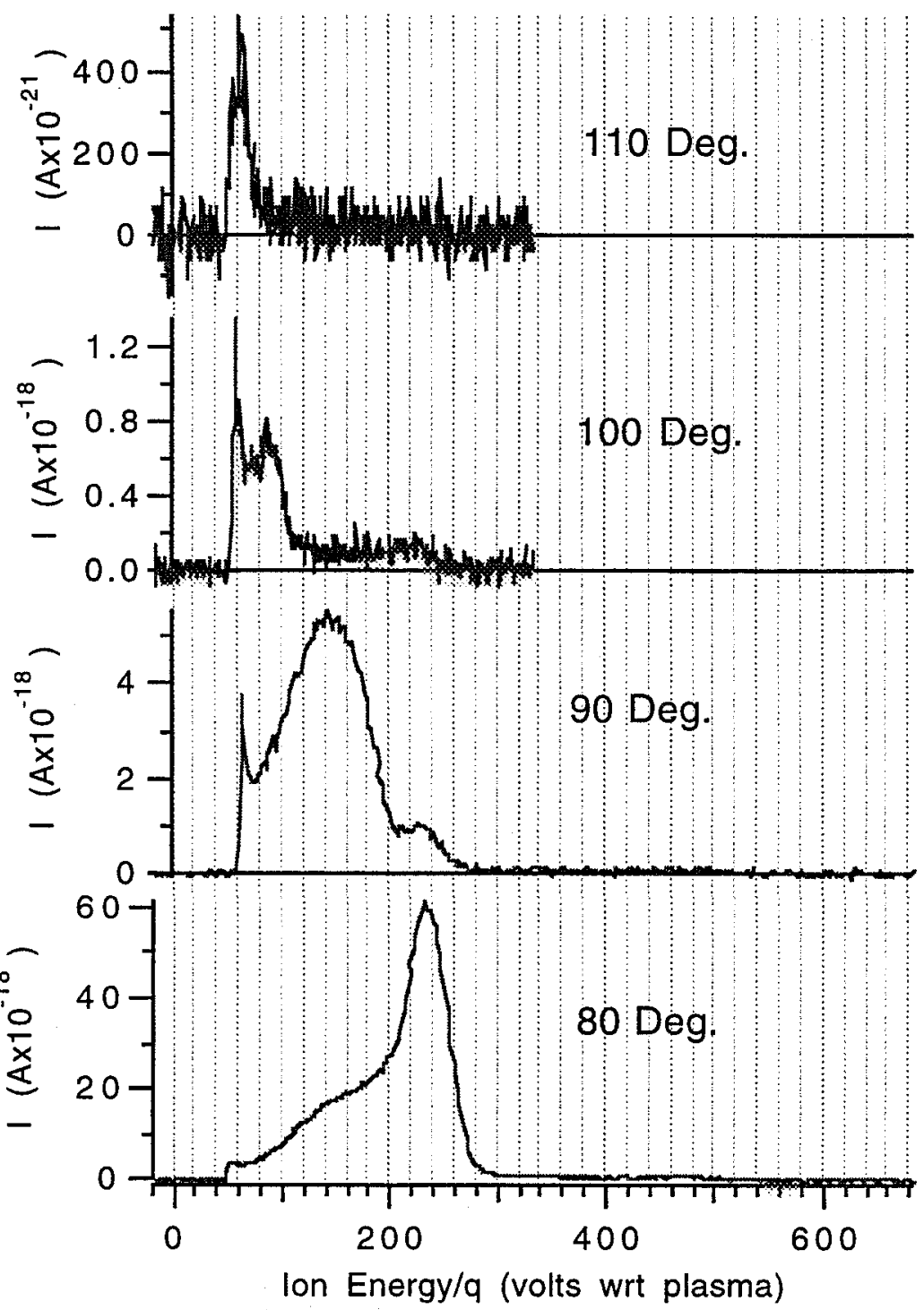

Figure 14. Ion current as a function of ion voltage at $1.0 \mathrm{~m}$ radius from the SPT-100 for points at $80,90,100$, and 110 degrees off thrust axis. 

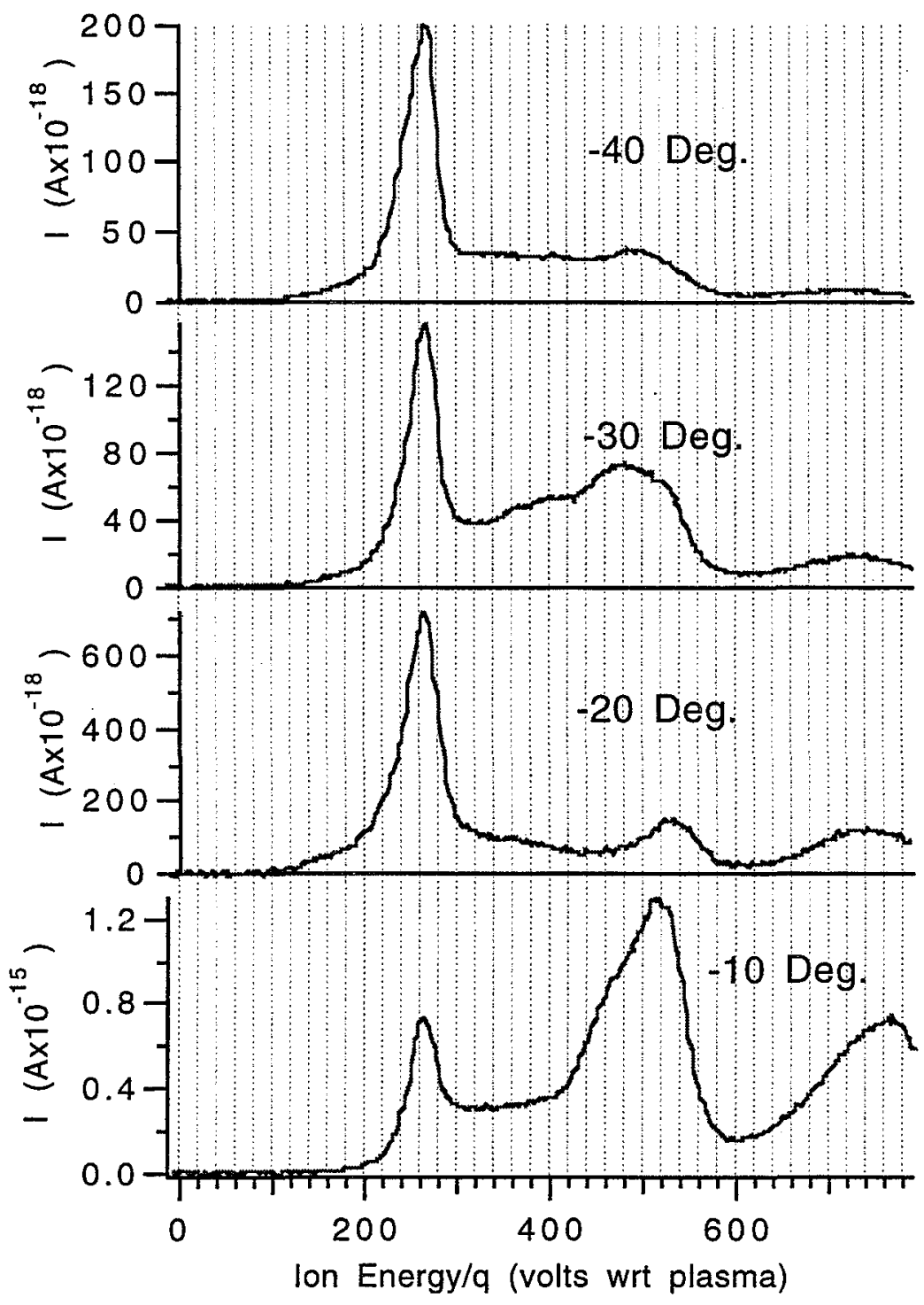

Figure 15. Ion current as a function of ion voltage at $1.0 \mathrm{~m}$ radius from the SPT-100 for points at $10,-20,-30$, and -40 degrees off thrust axis. 


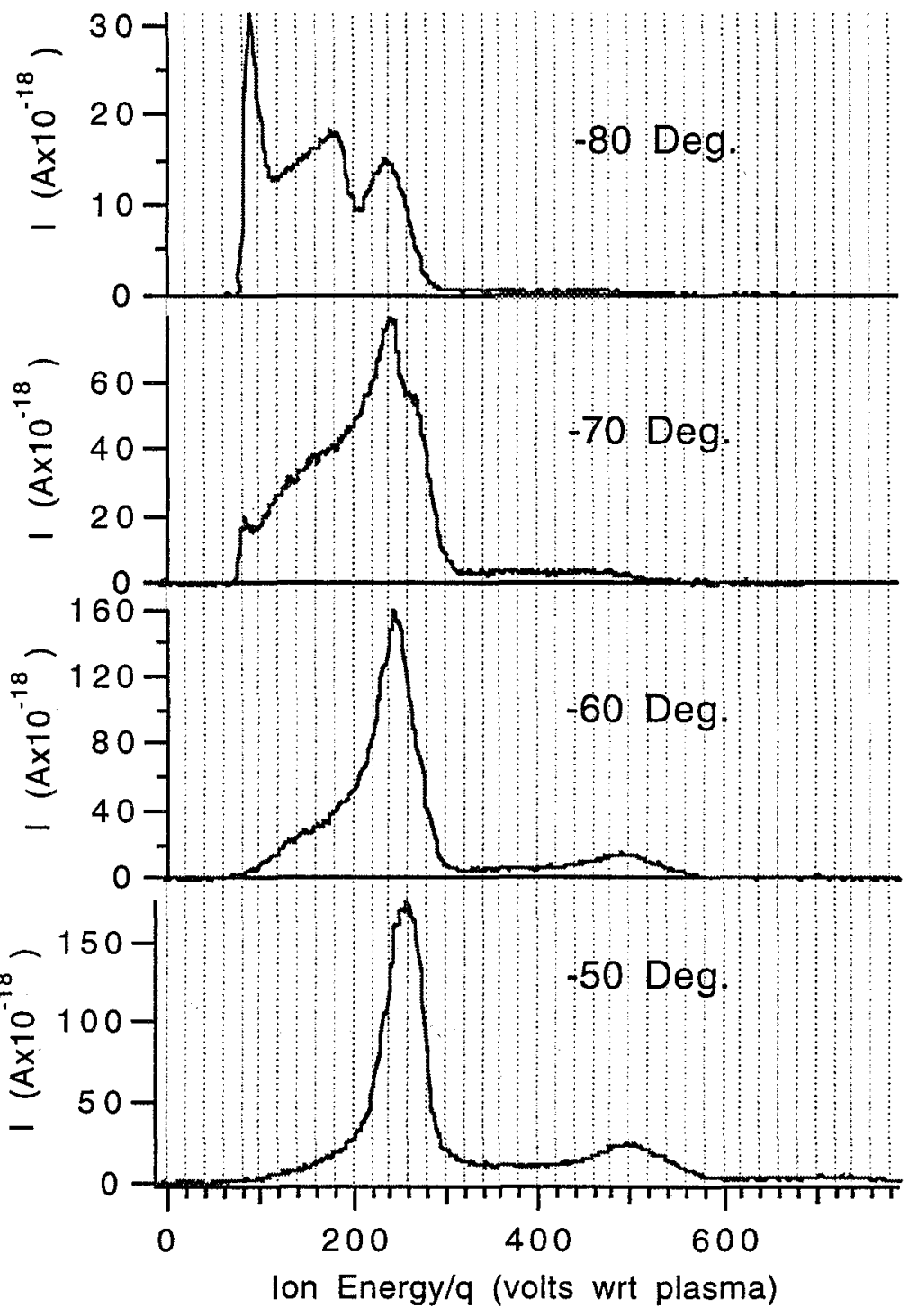

Figure 16. Ion current as a function of ion voltage at $1.0 \mathrm{~m}$ radius from the SPT-100 for points at $50,-60,-70$, and -80 degrees off thrust axis. 


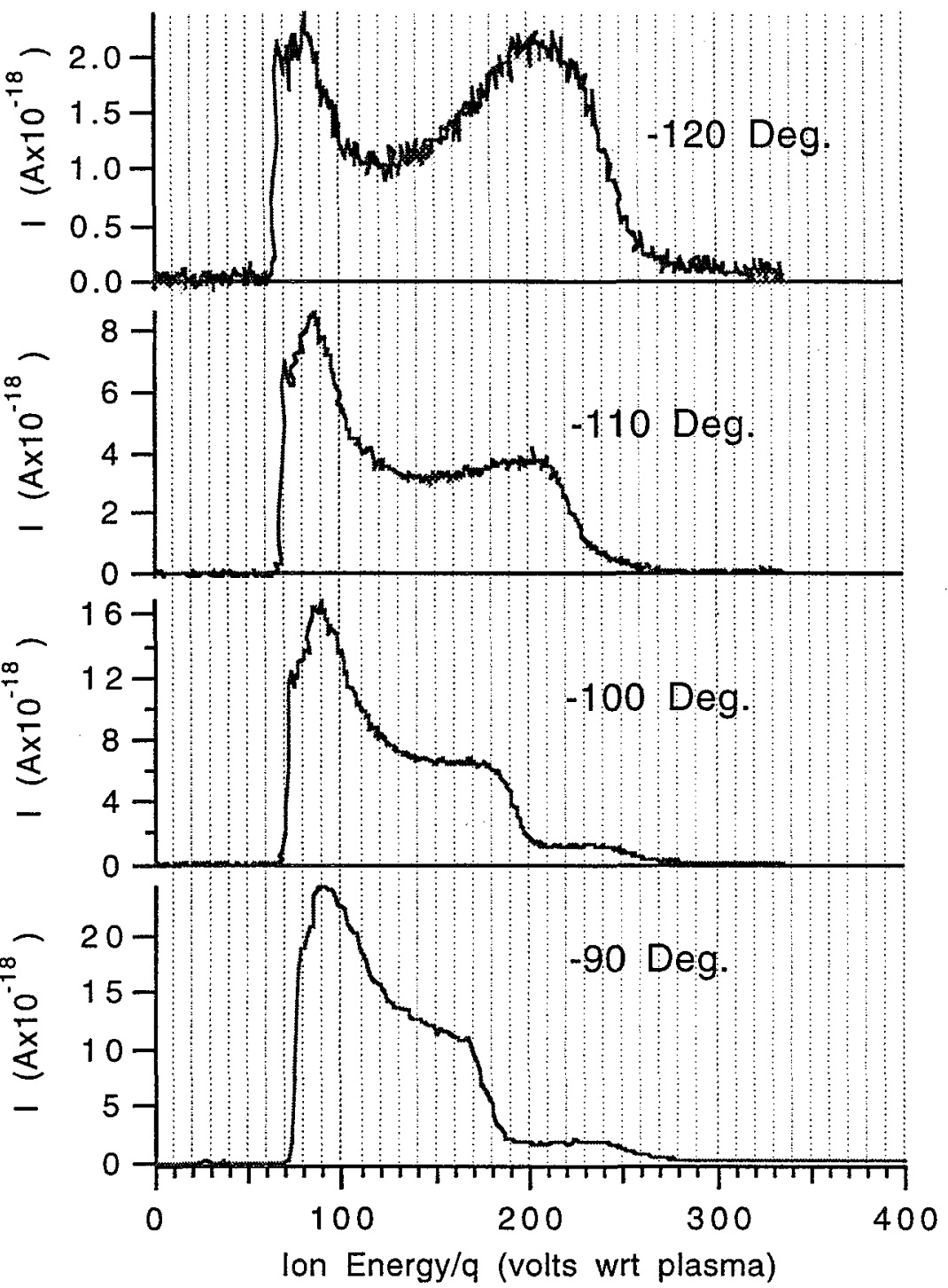

Figure 17. Ion current as a function of ion voltage at $1.0 \mathrm{~m}$ radius from the SPT-100 for points at $90,-100,-110$, and -120 degrees off thrust axis. 

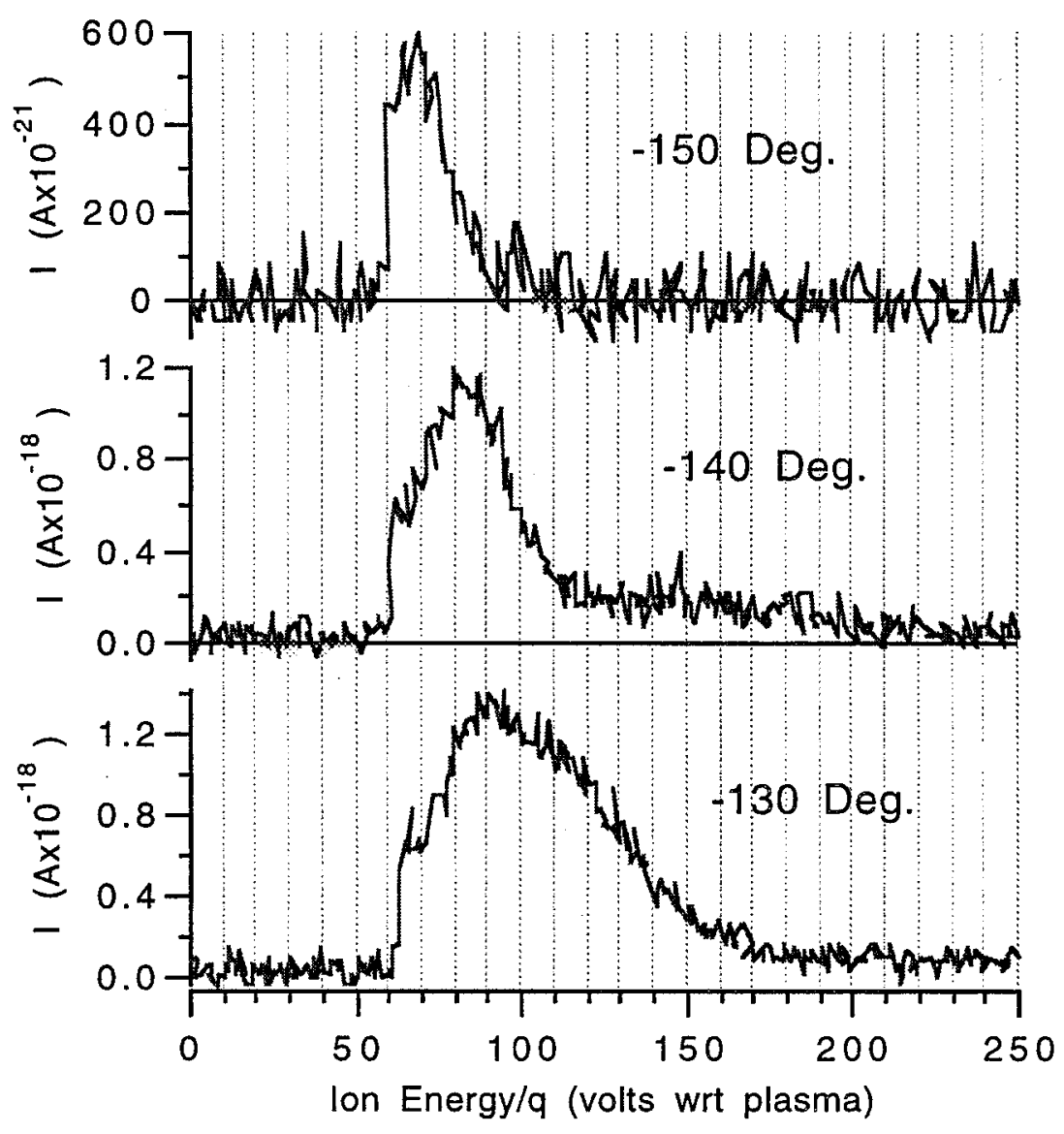

Figure 18. Ion current as a function of ion voltage at $1.0 \mathrm{~m}$ radius from the SPT-100 for points at $130,-140$, and -150 degrees off thrust axis. 


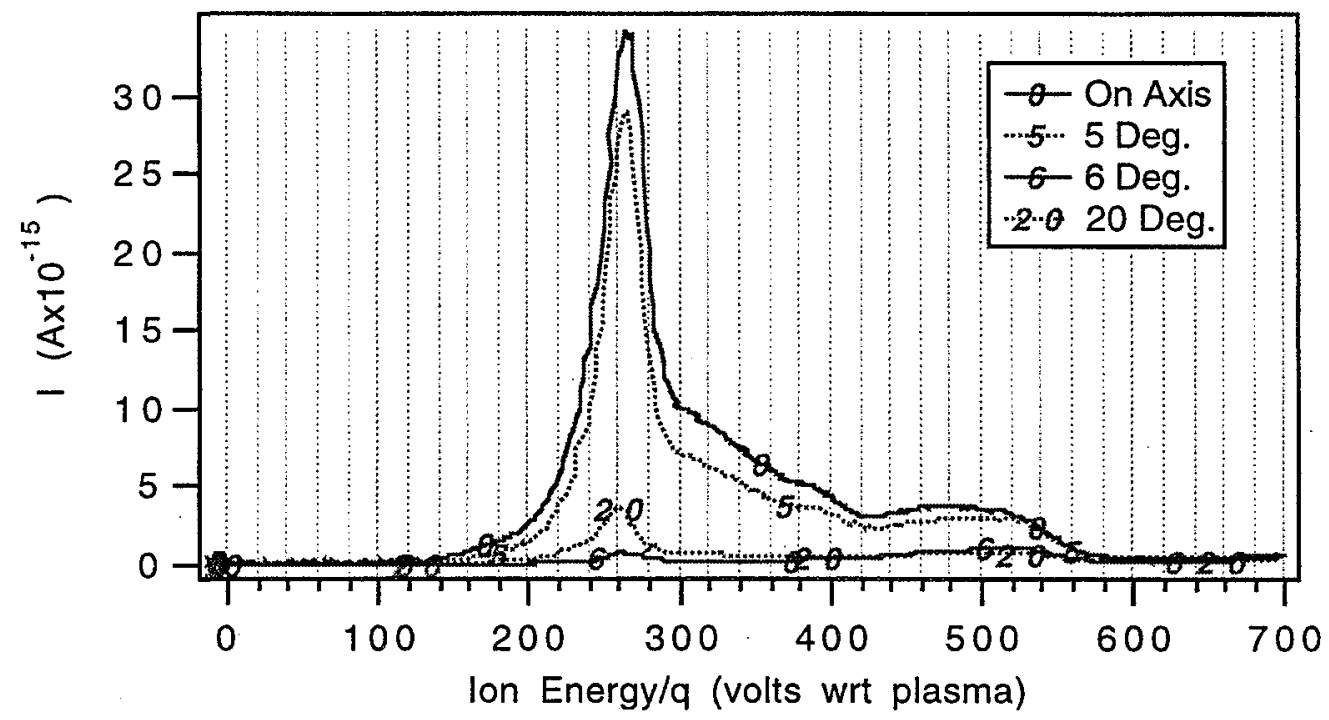

Figure 19. Evolution of ion current traces as a function of angular position between thrust axis and 20 degrees at $1.0 \mathrm{~m}$ radius from the SPT-100. 

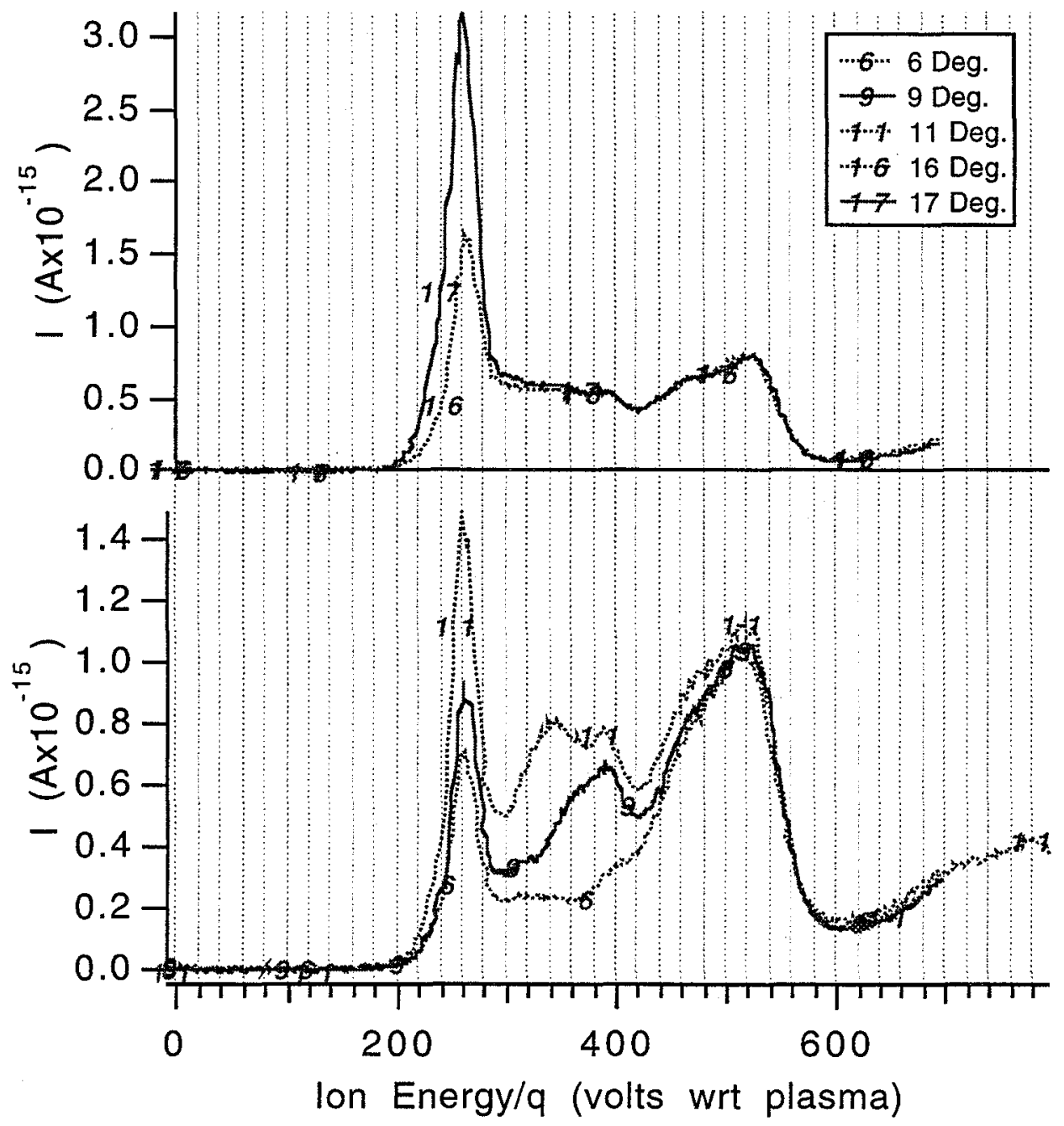

Figure 20. Exploded view of ion current evolution between 6 degrees and 17 degrees off thrust axis at $1.0 \mathrm{~m}$ radius from the SPT- 100 . 


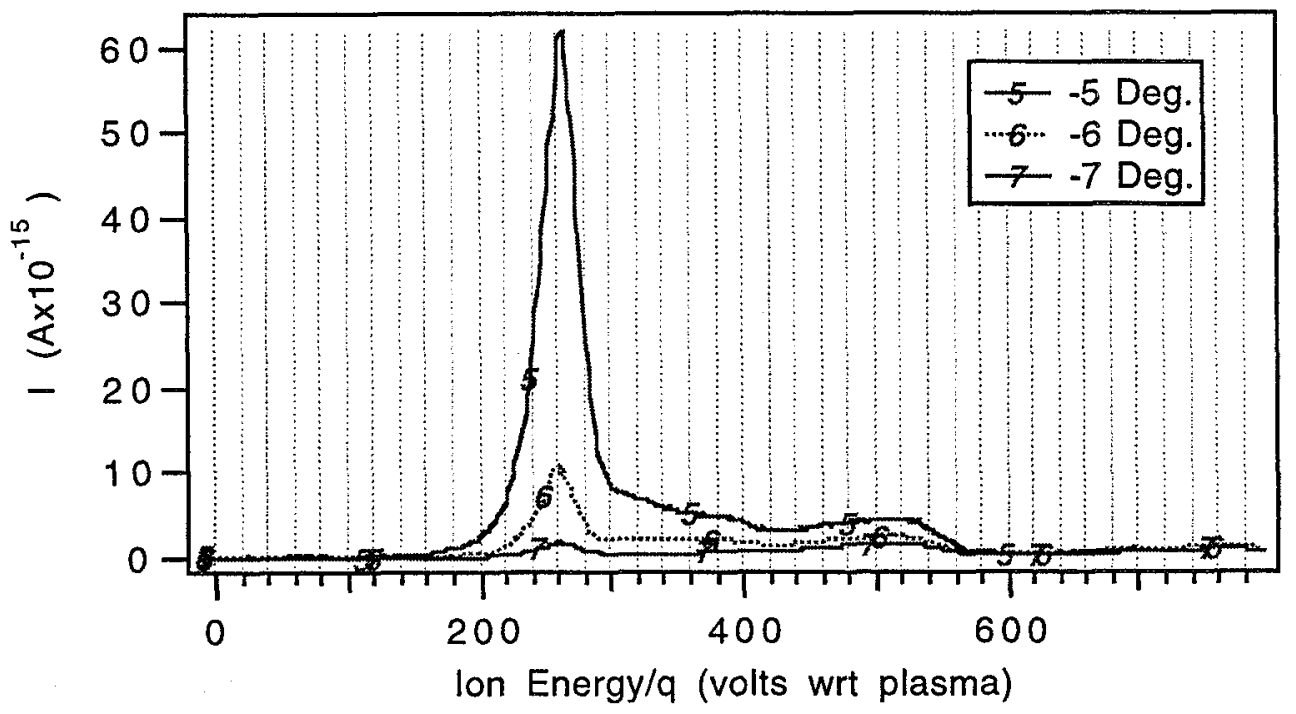

Figure 21. Ion current evolution between -5 and -7 degrees off thrust axis at $1.0 \mathrm{~m}$ radius from SPT-100. 


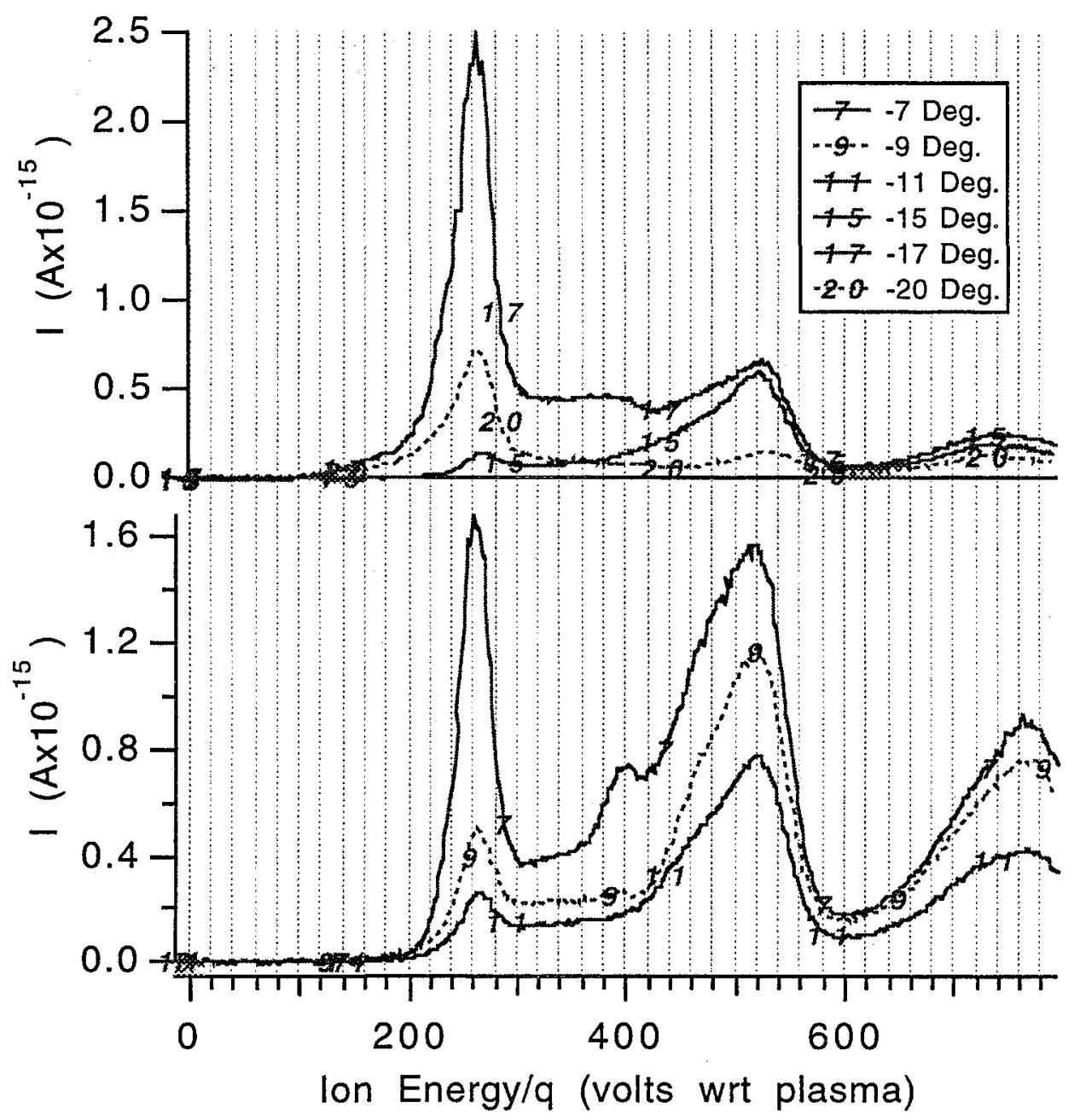

Figure 22. Ion current evolution between -7 degrees and -20 degrees off thrust axis at $1.0 \mathrm{~m}$ radius from the SPT-100. 


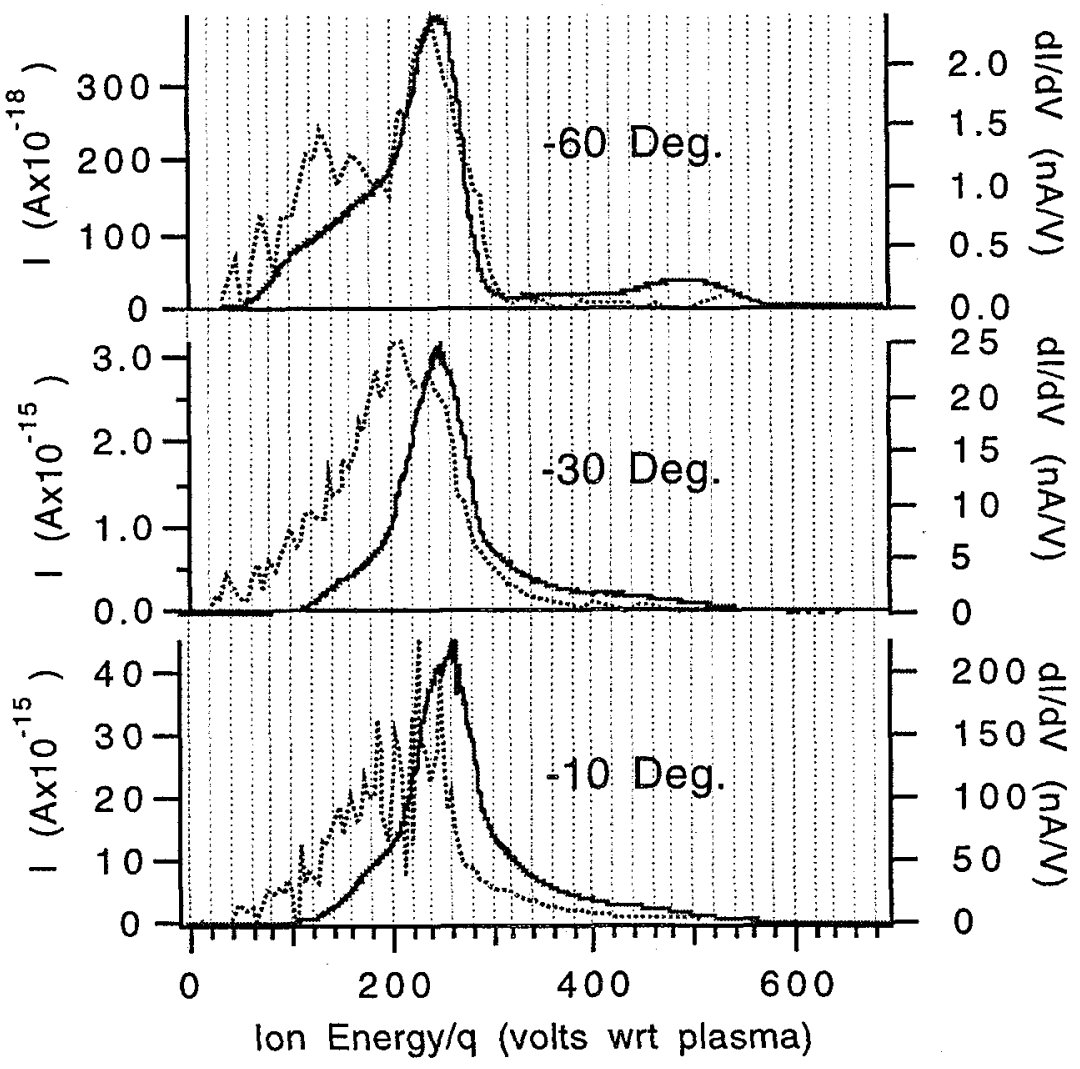

Figure 23. Comparison between RPA and MBMS measurements of the ion voltage at $0.5 \mathrm{~m}$ radius from the SPT-100. RPA traces are shown as dashed lines corresponding to the right vertical axes, with the MBMS data shown solid with units on the left axes. 


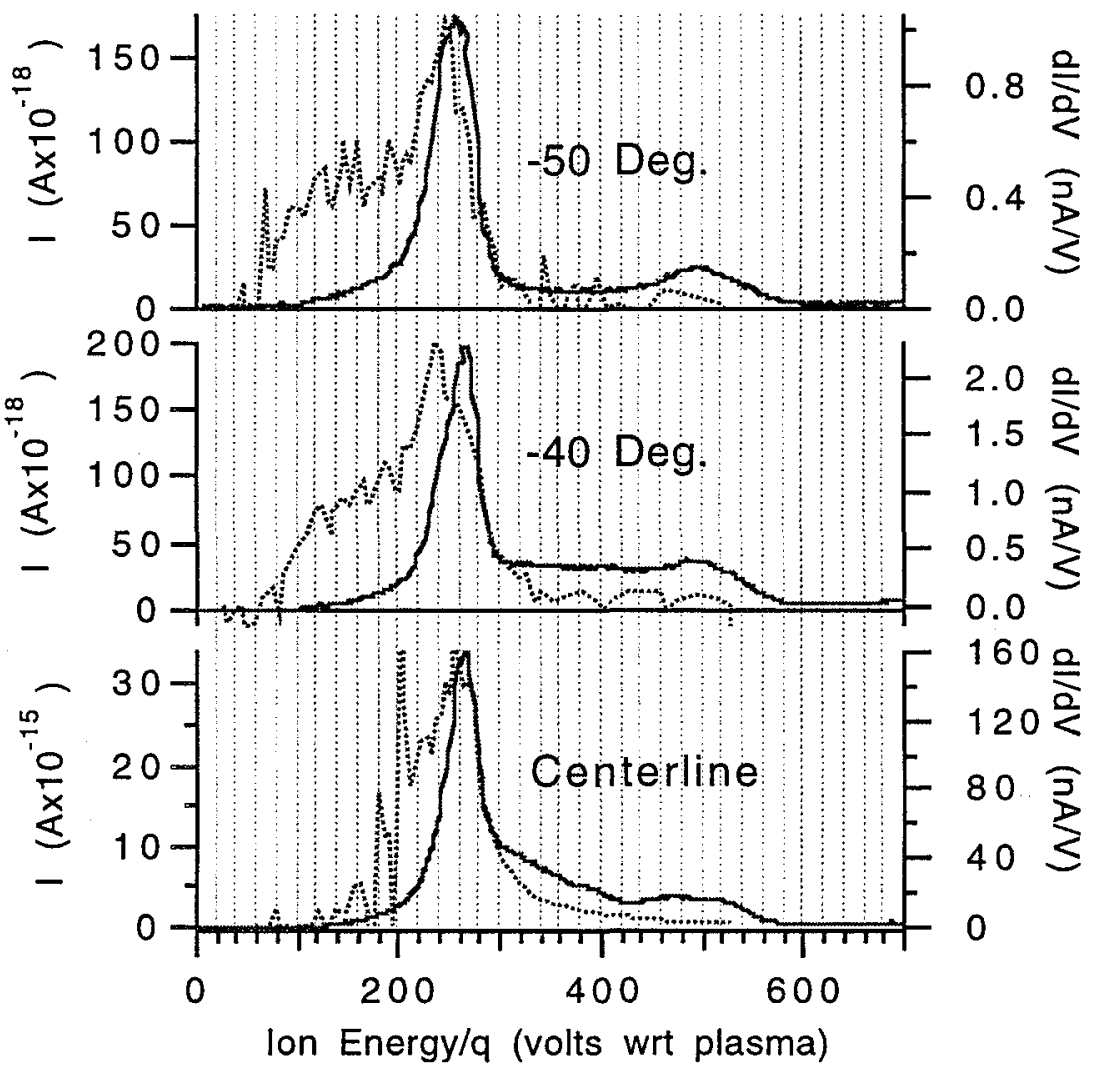

Figure 24. Comparison between RPA and MBMS measurements of the ion voltage at $1.0 \mathrm{~m}$ radius from the SPT-100. RPA traces are shown as dashed lines corresponding to the right vertical axes, with the MBMS data shown solid with units on the left axes. 


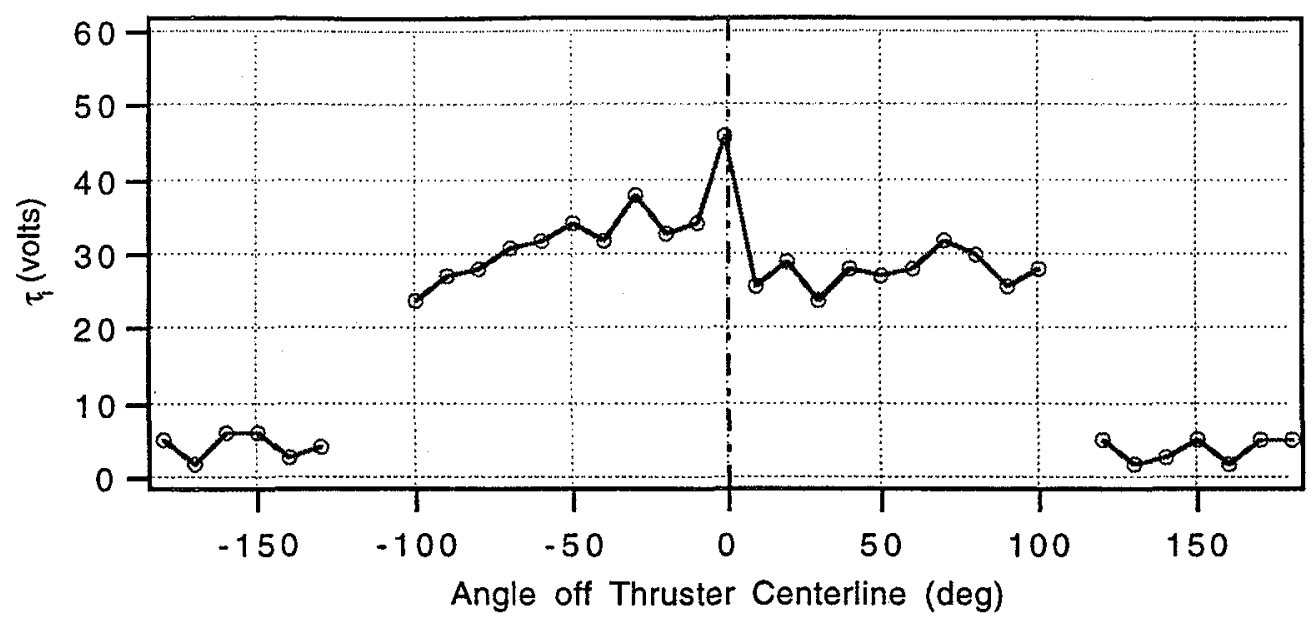

Figure 25. Variation of defined ion temperature, $\tau_{\mathrm{i}}$, as a function of angular position at $0.5 \mathrm{~m}$ radius from the SPT- 100 .

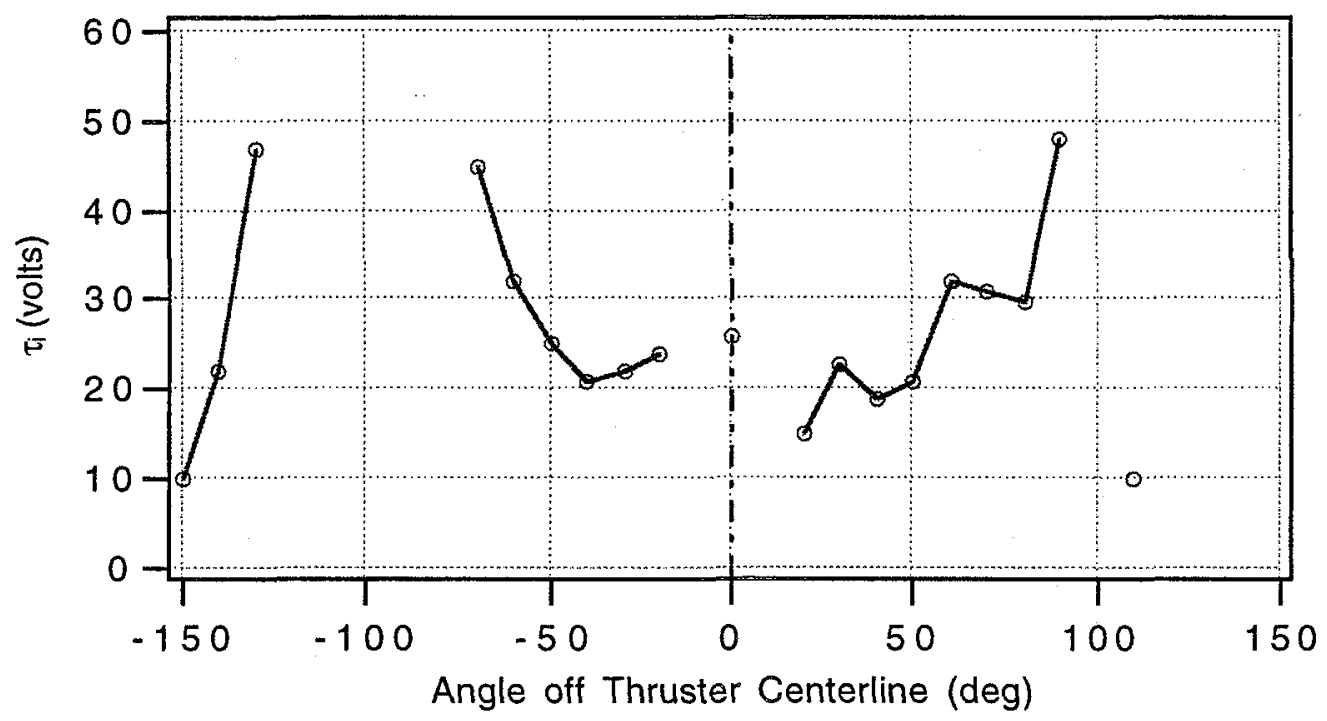

Figure 26. Variation of defined ion temperature, $\tau_{\mathrm{i}}$, as a function of angular position at $1.0 \mathrm{~m}$ radius from the SPT- 100 . 

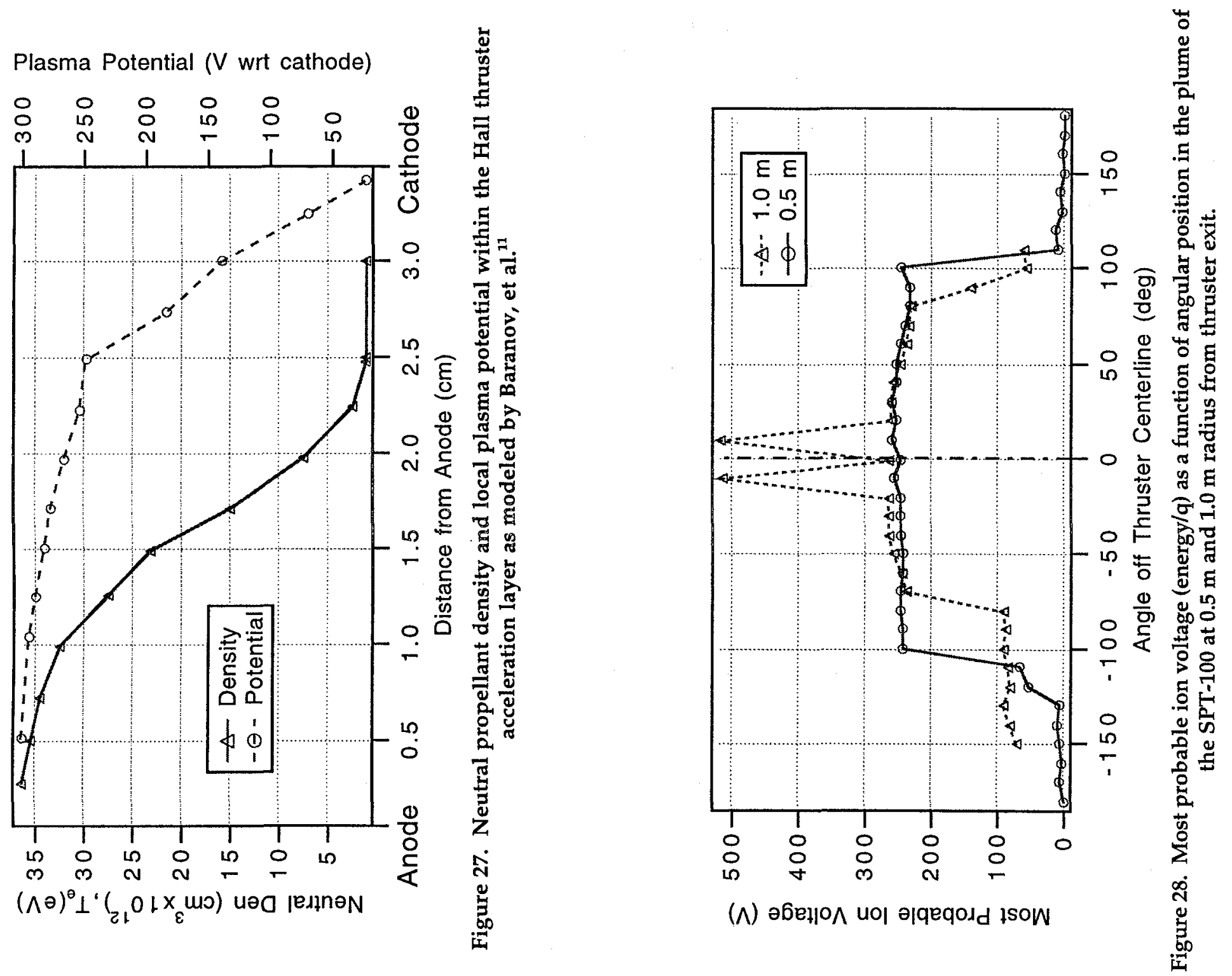


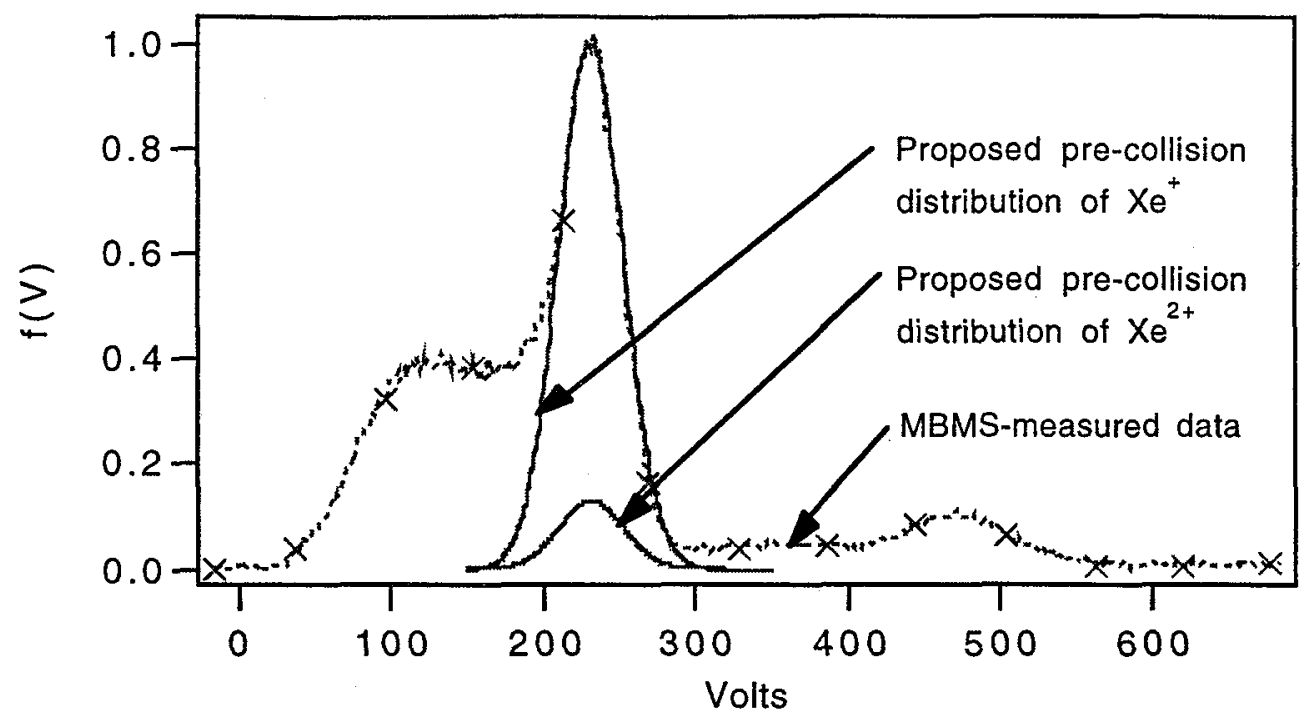

Figure 29. Proposed pre-collision gaussian distributions for $\mathrm{Xe}^{+}$and $\mathrm{Xe}^{2+}$ for the data at $0.5 \mathrm{~m}$ from the SPT-100, 90 degrees off axis.

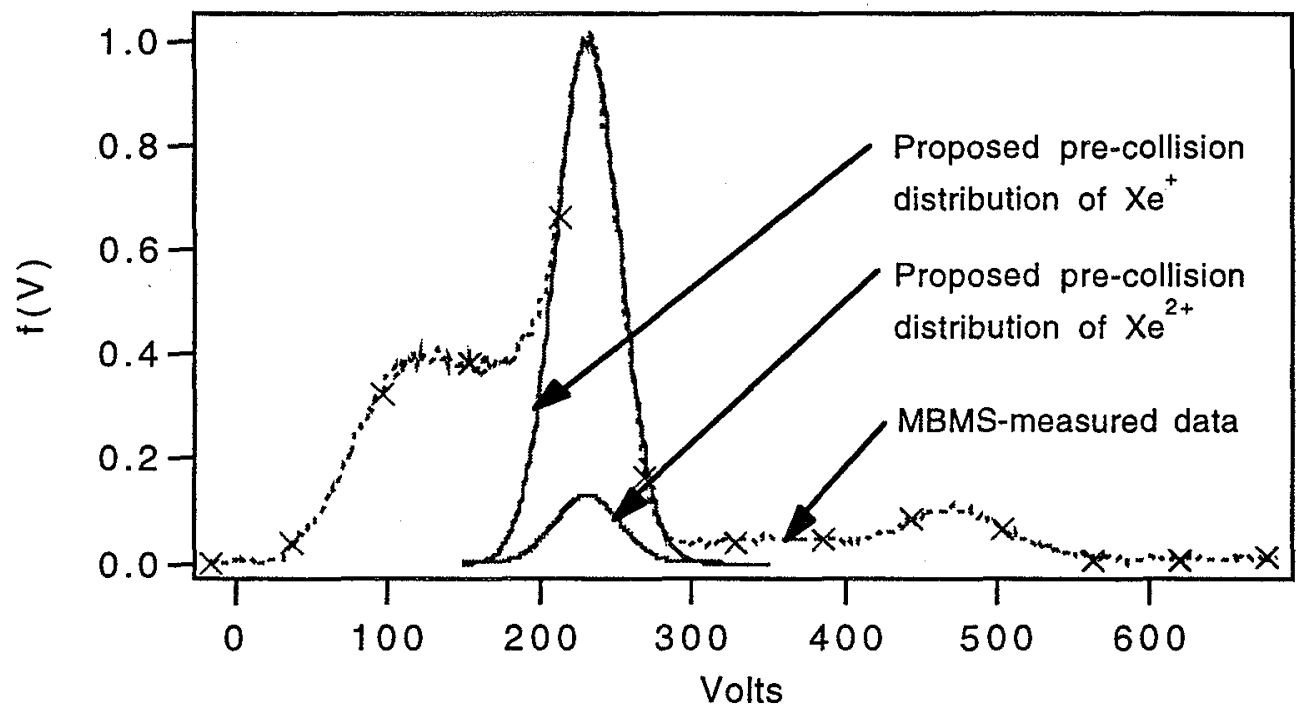

Figure 30. Proposed pre-collision gaussian distributions for $\mathrm{Xe}^{+}$and $\mathrm{Xe}^{2+}$ for the data at $0.5 \mathrm{~m}$ from the SPT-100, 90 degrees off axis. 


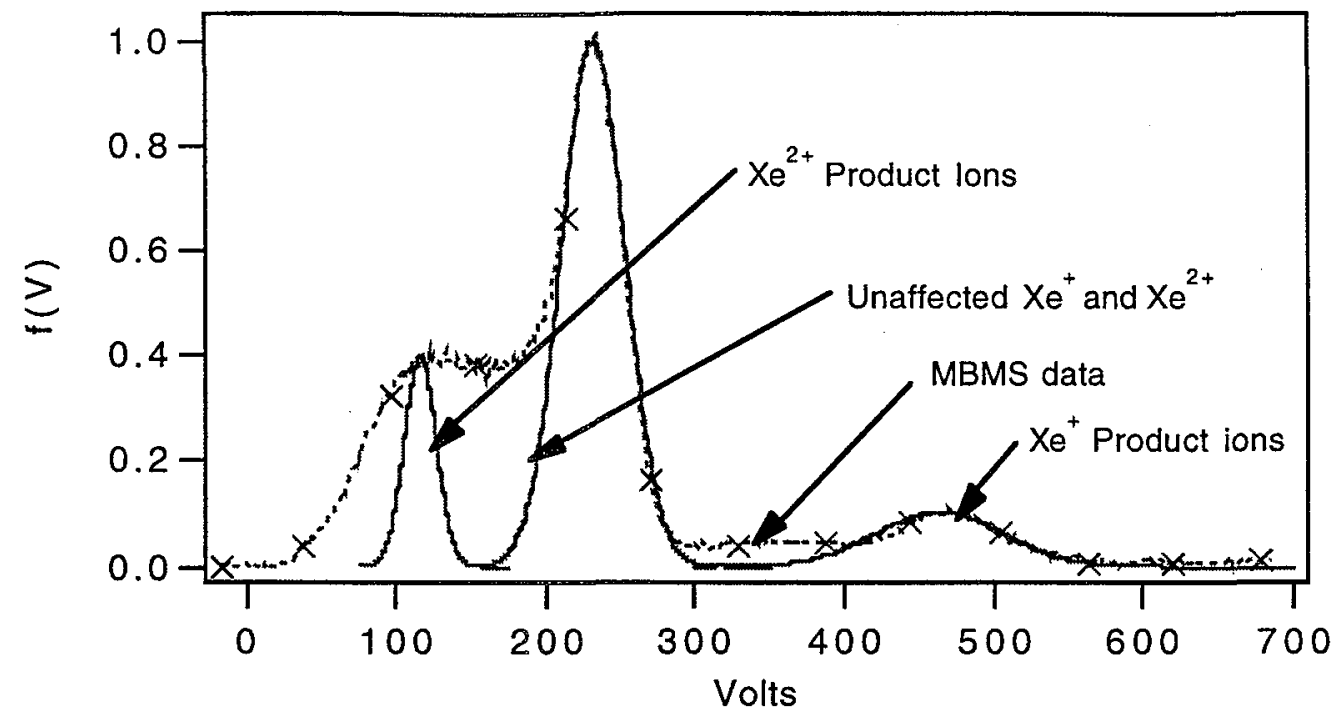

Figure 31. Post-collision distribution result of CE reaction between singly and doubly charged beam ions based on assumed gaussian pre-collision distribution.

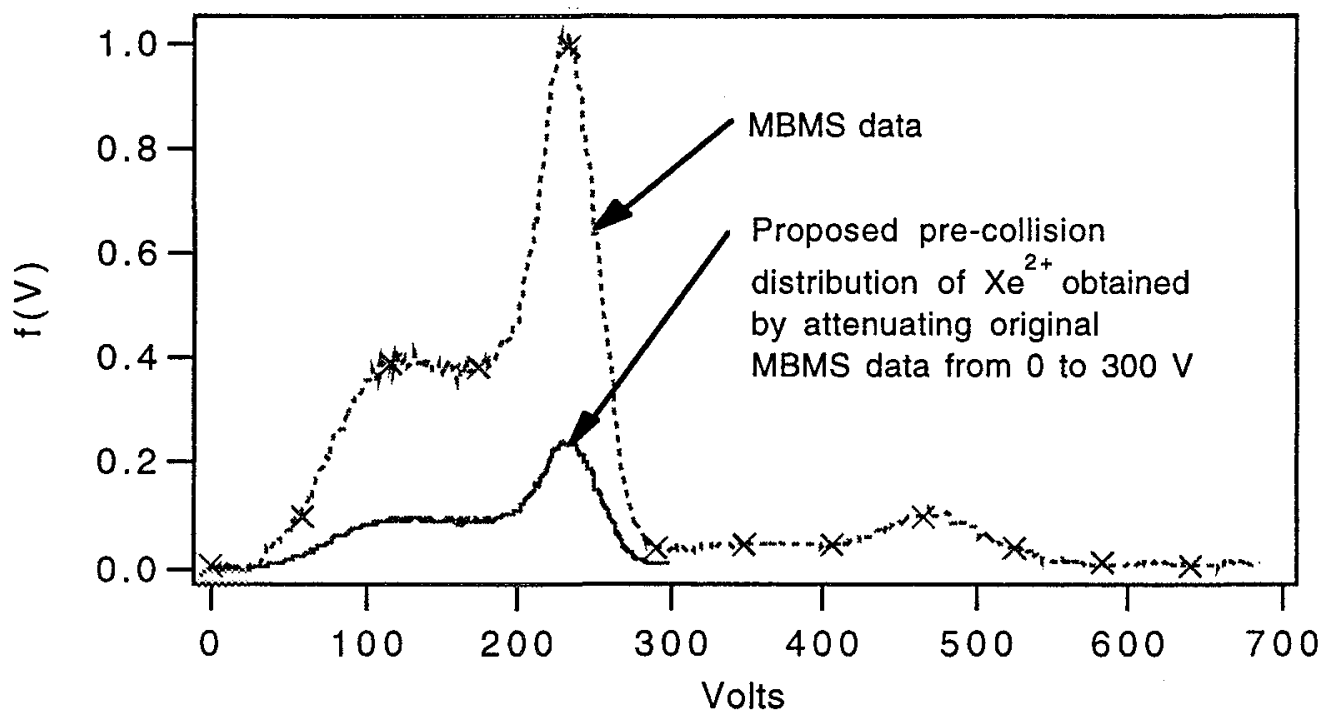

Figure 32. Proposed pre-collision distribution of $\mathrm{Xe}^{2+}$ computed from the MBMS data at $0.5 \mathrm{~m}, 90$ degrees off axis in the SPT-100. The proposed distribution was calculated as a fraction of the original data between 0 and $300 \mathrm{~V}$. 


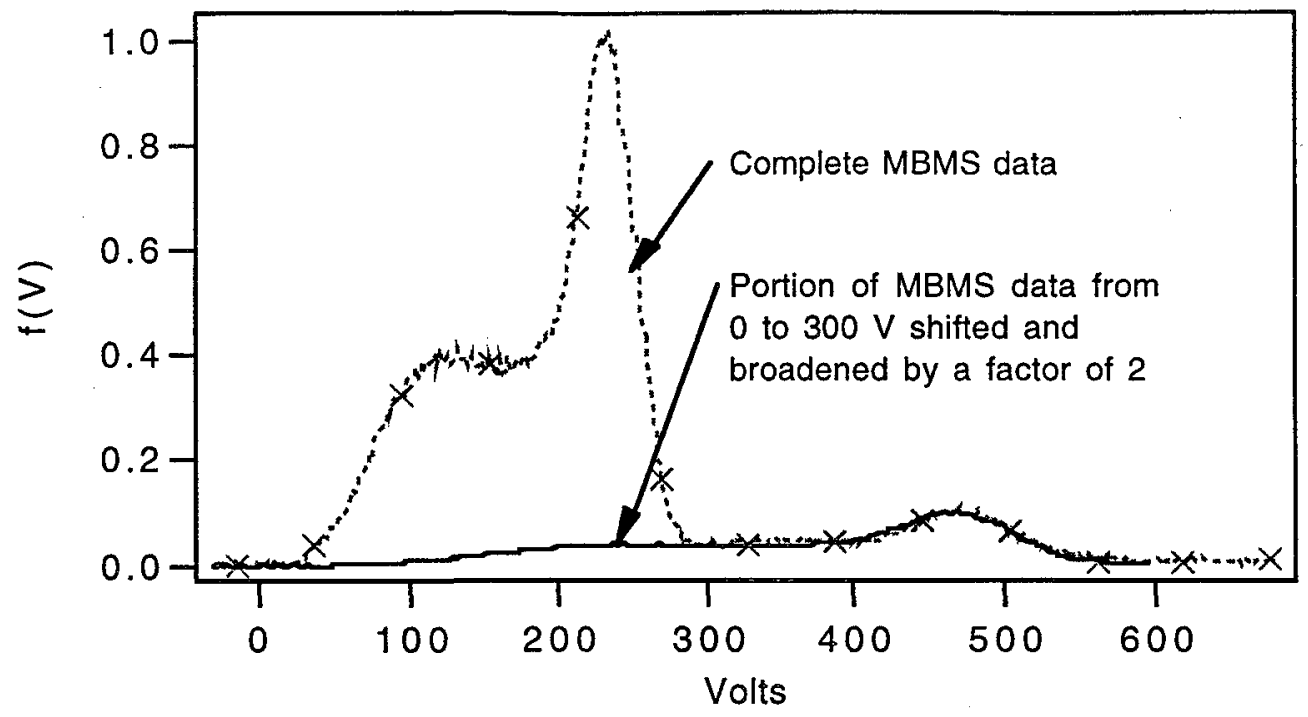

Figure 33. Illustration of identical shape in tail and main body of voltage distribution data measured at $0.5 \mathrm{~m}$ radius and 90 degrees off centerline in the SPT-100.

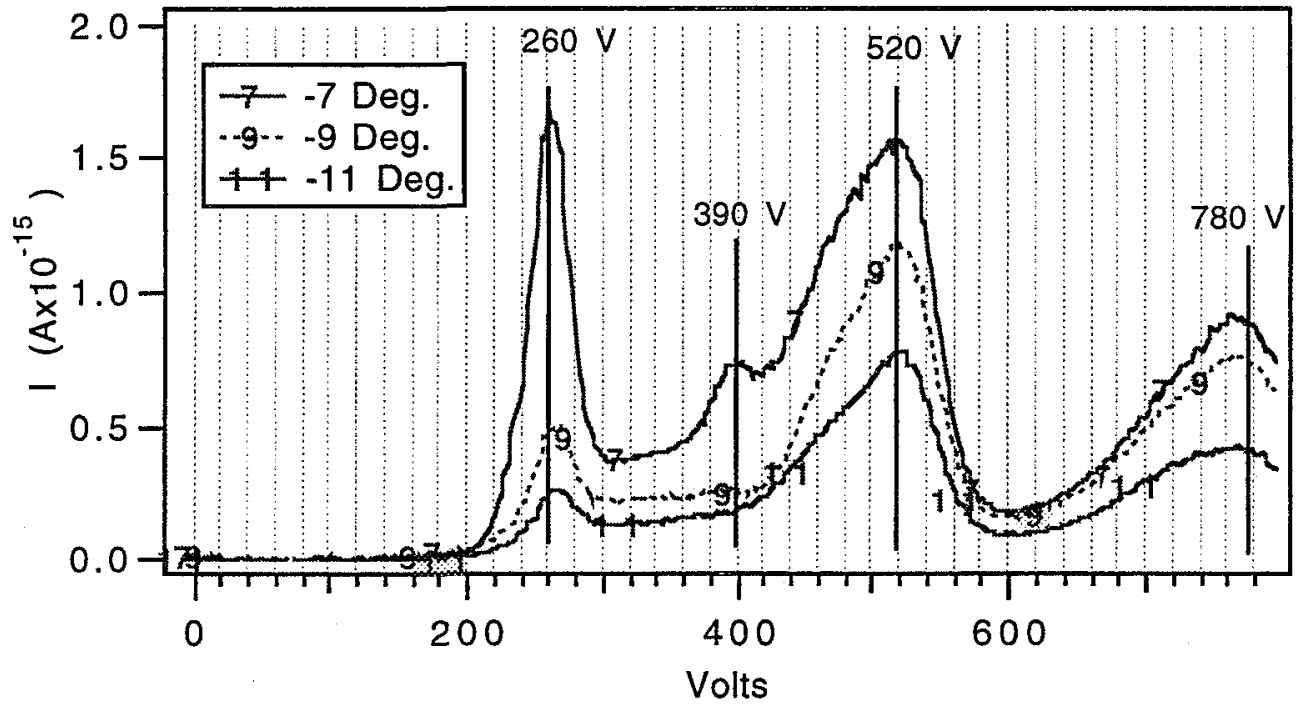

Figure 34. Ion energy distribution measured at $1.0 \mathrm{~m}$ radius from the SPT-100 for angles at $-7,-9$, and -11 degrees off thrust axis. Clearly evident are the high-voltage peaks at $3 V_{b} / 2,2 V_{b}$, and $3 \mathrm{~V}_{\mathrm{b}}$ produced as a result of $\mathrm{CE}$ collisions with neutral atoms. 


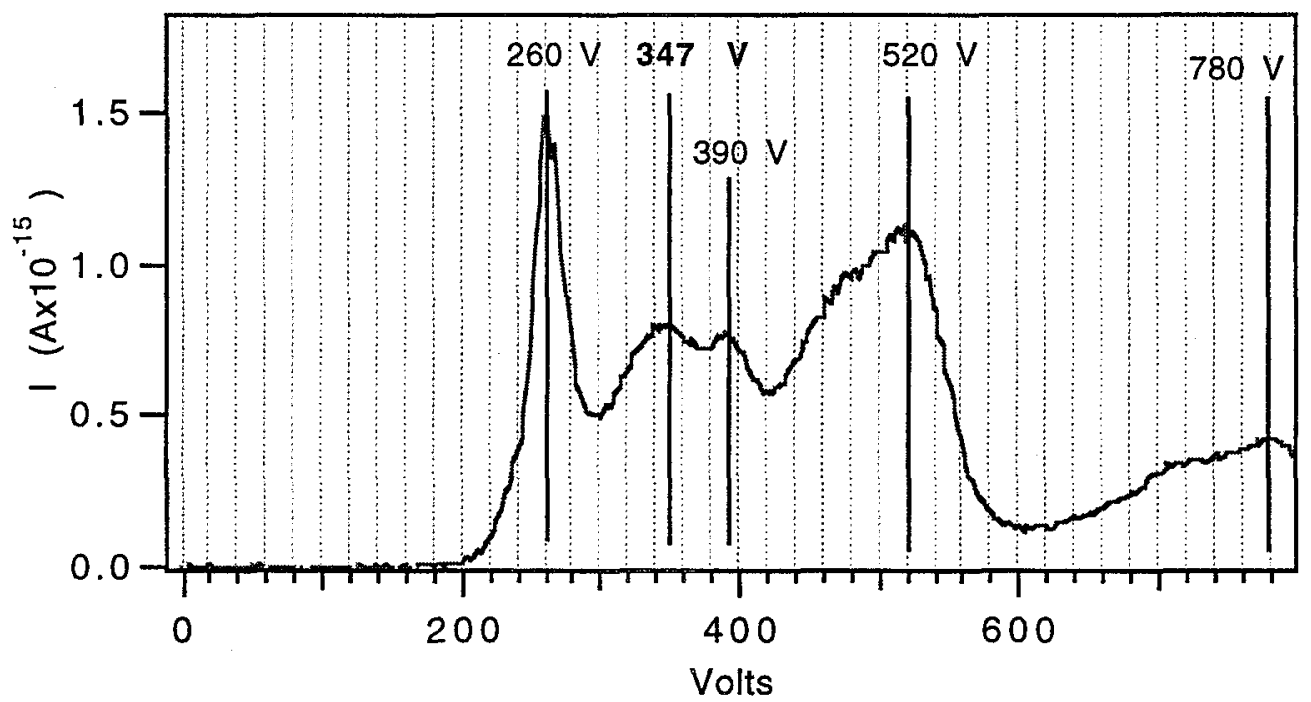

Figure 35. Ion voltage distribution at $1.0 \mathrm{~m}$ radius from the SPT-100 plume at 11 degrees off axis showing peaks at $4 \mathrm{~V}_{\mathrm{b}} / 3,3 \mathrm{~V}_{\mathrm{b}} / 2,2 \mathrm{~V}_{\mathrm{b}}$, and $3 \mathrm{~V}_{\mathrm{b}}$. 